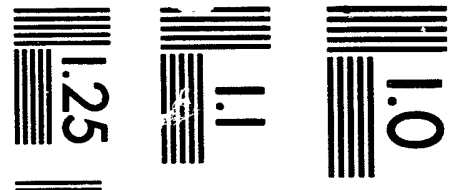

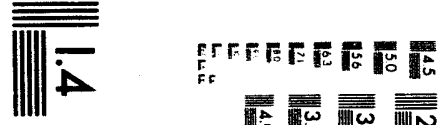

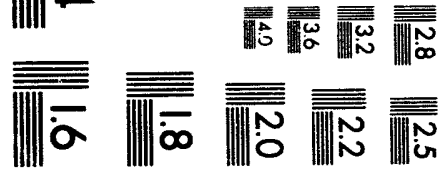



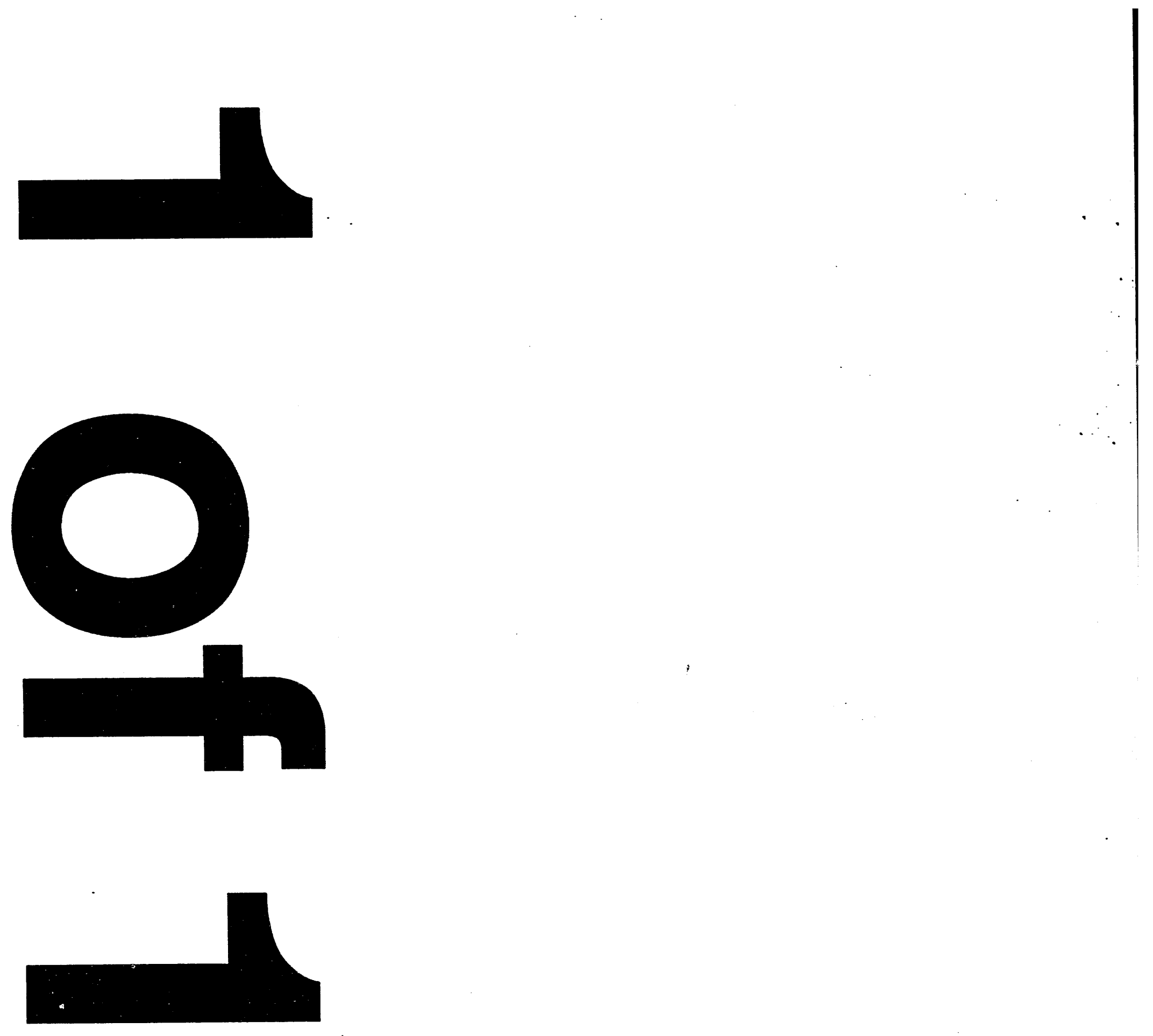
Directional Measurements

for Sources of Fission Neutrons

Roger C. Byrd

George F. Auchampaugh

William C. Feldman 


\title{
DIRECTIONAL MEASUREMENTS FOR SOURCES OF FISSION NEUTRONS
}

by

Roger C. Byrd, George F. Auchampaugh, and William C. Feldman

\begin{abstract}
Although penetrating neutron and gamma-ray emissions arguably provide the most effective signals for locating sources of nuclear radiation, their relatively low fluxes make searching for radioactive materials a tedious process. Even assuming lightly shielded sources and detectors with large areas and high efficiencies, estimated counting times can exceed several minutes for source separations greater than ten meters. Because determining the source position requires measurements at several locations, each with its own background, the search procedure can be lengthy and difficult to automate. Although directional measurements can be helpful, conventional collimation reduces count rates and increases the detector size and weight prohibitively, especially for neutron instruments.

We describe an alternative approach for locating radiation sources that is based on the concept of a polarized radiation field. In this model, the presence of a source adds a directional component to the randomly oriented background radiation. The net direction of the local field indicates the source angle, and the magnitude provides an estimate of the distance to the source. The search detector is therefore seen as a device that responds to this polarized radiation field. Our proposed instrument simply substitutes segmented detectors for conventional single-element ones, so it requires little or no collimating material or additional weight. Attenuation across the detector creates differences in the count rates for opposite segments, whose ratios can be used to calculate the orthogonal components of the polarization vector. Although this approach is applicable to different types of radiation and detectors, in this report we demonstrate its use for sources of fission neutrons by using a prototype fast-neutron detector, which also provides background-corrected energy spectra for the incident neutrons. Computer simulations of possible search procedures indicate that the directional capability should significantly improve the performance of searches for nuclear sources.
\end{abstract}




\section{INTRODUCTION}

Shielding for Radioactive Materials. Because many nuclear weapons are based on the radioactive material plutonium, one of the clearest signatures for their detection is the penetrating radiation of $\mathrm{x}$-rays, gamma rays, and neutrons that they emit. High-density materials such as lead can be used to shield the two electromagnetic radiations, although large thicknesses may be needed for gamma rays at high energies. Shielding for fission neutrons, on the other hand, requires bulky but relatively lightweight hydrogenous materials such as plastic or water. Effective shielding for both neutrons and gamma rays must therefore be large and massive, so a well-shielded shipping container would be suspicious because of its sheer size and weight. Nuclear search techniques usually exploit this difficulty by utilizing both types of detectors.

Directional Radiation Detectors. For portable instruments, shielding constraints also affect the design of collimators for determining the location of a radiation source. Because modest amounts of lead can provide significant attenuation at most energies, fielding a directional gamma-ray detector poses little difficulty. Effective collimation for fast neutrons is much more cumbersome, so neutron detectors are usually deployed without directionality. For long-range searches, the large size of any detector makes effective shielding impossible. Thus, it would be useful to develop techniques for determining the direction of incident radiation that would be effective even with unshielded detectors, particularly those used for fast neutrons.

Related Work. A recent report ${ }^{1}$ discusses computer simulations of directionality for shielded and unshielded fast-neutron detectors. The present paper continues this study by using actual measurements to examine some practical applications. The detector employed is a novel instrument originally developed as a neutron spectrometer for space applications. ${ }^{2}$ It has also been used for studying neutron-induced fission in enriched uranium, ${ }^{3}$ for counting warheads on silo-based missiles, ${ }^{4}$ and for measuring far-field fluxes from a nuclear reactor. ${ }^{5}$ The measurements are part of a study of fission spectra for realistic mockups of nuclear weapons. A companion paper ${ }^{6}$ describes the detector calibration and spectral analysis, and a classified report will discuss specific features of the mockup spectra. Although the present measurements were not originally intended to demonstrate the detector's directionality, they nevertheless provide an extensive and realistic set of test cases.

Contents. Section 2 presents a brief summary of the operating principles of the unique detector used in the present work, which is described more fully in Refs. 2 and 6 . Section 3 explains the concepts of a directional radiation field and its characterization using a multielement detector. Section 4 presents the mathematical formalism for extracting the directional information from the measured count rates, and it contains the bulk of the measurements that demonstrate the basic technique. Section 5 uses Monte Carlo simulations to explore the advantages that the directional capability can provide in hypothetical search scenarios. Section 6 describes two additional applications of segmented neutron detectors. One extends the directional analysis to the estimation of source separation distances, and the other discusses a technique proposed in Ref. 1 for obtaining background corrections to measured energy spectra. Section 7 summarizes the results from the entire report.

\section{DETECTOR OPERATION}

Proton Recoil Scintillators. In organic scintillation detectors, neutrons that scatter from protons in the plastic produce proton-recoil energy and detectable light output. Most neutrons scatter only a few times and then escape, so they lose only a fraction of their original energy. Some, however, scatter through large enough angles to deposit essentially 
all their energy in the first few collisions, and they become trapped in the scintillator. Because of saturation in the scintillator's energy-to-light conversion at energies below $0.5 \mathrm{MeV}$ (see Ref. 6), further collisions do not provide additional signals. Thus, almost all the energy deposited by a trapped neutron is collected into a single prompt proton-recoil pulse, whose amplitude is directly related to the incident neutron energy. Unfortunately, with a conventional scintillator there is no way to distinguish between signals from trapped and escaping neutrons, so a continuous distribution of proton-recoil energies is obtained. The energy spectrum of the original neutrons can be recovered only by unfolding this complex distribution.

Boron-Loaded Spectrometers. The first unusual feature of the detector used in the present experiment is its capability to identify those fast neutrons that stop in the detector. As described in Refs. 2 and 6, the active element is a plastic scintillator (Bicron BC454) that has been loaded to $1 \%$ by weight with ${ }^{10} \mathrm{~B}$. As in a conventional scintillator, neutrons that scatter once or twice and then escape produce a single recoil pulse; those that deposit almost all their energy usually remain in the scintillator. In our case, within a few $\mu$ s many of these trapped neutrons interact with the added boron through the ${ }^{10} \mathrm{~B}(\mathrm{n}, \alpha){ }^{7} \mathrm{Li}$ capture reaction, which produces a second light pulse. Thus, observing a rapid sequence of two pulses, the first from the energetic proton recoils and the second from the boron capture, provides a means of selecting only those incident neutrons that (1) have original energies above $0.5 \mathrm{MeV}$ and (2) deposit essentially all their energy in the recoil pulse. In these cases the spectrum of proton recoils is also the energy spectrum of the incident neutrons-but without requiring any unfolding. These energy distributions are discussed in detail in Ref. 6; in this report they will figure mainly in the development of the background corrections in Sec. 6.

Multielement Detector Directionality. The second unusual feature of the detector is the one most important for the present work. As shown in Fig. 2.1, the instrument head consists of four boron-loaded scintillator rods with photomultiplier tubes at each end. The four side-by-side scintillator elements are intended to indicate the direction of the incident neutron flux. In the original description, ${ }^{2}$ this angular capability was demonstrated only for charged particles (muons generated by cosmic-ray interactions with the spacecraft). As developed in Ref. 1, the directional information can be used in two other ways. First, differences in solid angle and attenuation across the detector create differences between the count rates for different segments, which can be used to calculate the direction of the incident flux. The calculations suggest that the systematic angle accuracy for an unshielded detector should probably be within $10^{\circ}$. Second, for sources placed in front of one pair of rods (as in this experiment), the opposite pair can be used to estimate a correction for room backgrounds. Under some circumstances, such a correction can provide an uncollimated detector with an essentially one-sided response.

Discussion. This brief description of the instrument used in the present measurements has stressed the two features most important for the present analyses. First, the scatter-andcapture selection restricts the response only to neutrons, only at energies above $0.5 \mathrm{MeV}$, and only those that deposit all their energy in the detector. Second, the four-element segmentation provides some information about the direction of the incident flux. Previously, this information has received only a cursory examination by means of simple computer simulations ${ }^{1}$ or measurements with charged particles, ${ }^{2}$ which behave very differently from the neutrons and gamma rays of interest for radiation search applications. Furthermore, the previous analysis ${ }^{1}$ focused on determining only source directions and background corrections. Neither of these applications is a central factor in the present experiment, which used a low-scattering cnvironment (so the background corrections are small) and sources placed directly in front of the detector (so the incident angles are known). Instead, the following analyses reveal that an important advantage of the directional information lies 


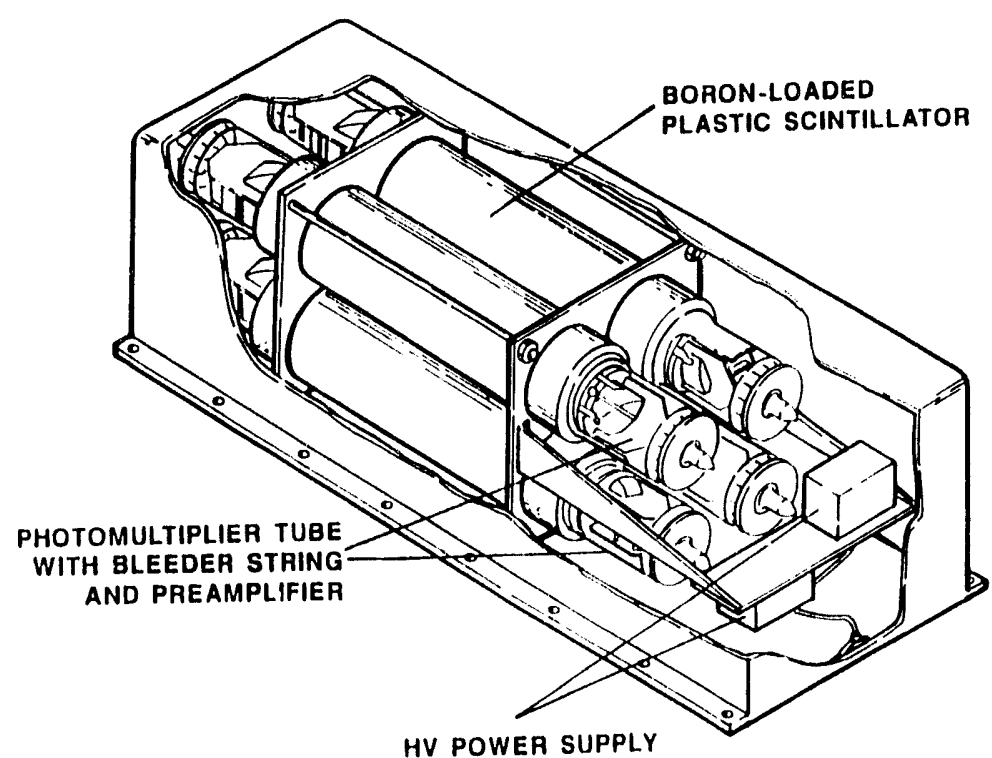

Fig. 2.1. (ntaway view of the nent "on detector. Four boron-loaded scintillator rods, cach with two photomulliplier tubes, provide information about the direction of the incident nentron flux. Most of thre clectronic components are contained in separate packages (not shown).

in a third application. which uses the differences between the count rates in different elements to indicate the existence, magnitude, and direction of a polarized radiation field. The development of this concept occupies most of the next section.

\section{POLARIZED RADIATION FIELDS}

Introduction. In a traditional search for a radiation source, count-rate measurements are made using a stambard grid spacing, and the signature of a nearby source is the inversesquare increase in thre mrasured flux as the sonce is approached. For distant sources this simple prescription is complicated by two additional factors. First, any radiation measurement includes random fluctuations from connting statistics, which create a fractional count-rate uncertainty that varies inversely as the square root of the number of counts: $\Delta N / N=1 / \sqrt{N}$. Sreond, all detectors respond to some degree to diffuse background radiation, whether natural or scattered from the source. This background contributes an obscuring count-rate variation that becomes increasingly important as the source flux decreases with the scpure of the separation. In principle, a systematic strategy could be developed to choose optimum step sizes and scarch directions from the accumulated countrate information. Instad, we will develop an additional signature of a radiation source that is based on the directional characteristics of the omited flux.

Source and Background Fluxes. Fo illustrate the essential features of the search pr blem, we have devised a computer simulation that incorporates the inverse-square falloff in the source flux, the resulting variations in the signal-1o-background ratio $(S / B)$, and the effects of counting statistics. Figure 3.1a illustrates the radial decrease in the flux from a $1 / r^{2}$ source (open circles) in the presence of a miform background (solid circles). The ratio between the 500 background counts and hor los signal counts is chosen to produce $S / B=5: 1$ at the indicated 5 -m source separation, which is the appropriate ratio for the measurements described in this report. Note that the continued $1 / r^{2}$ falloff in signal causes the $S / B$ ratio to approach $1: 1$ at a 10 -m soparalion. This figure indicates the difficulty of 


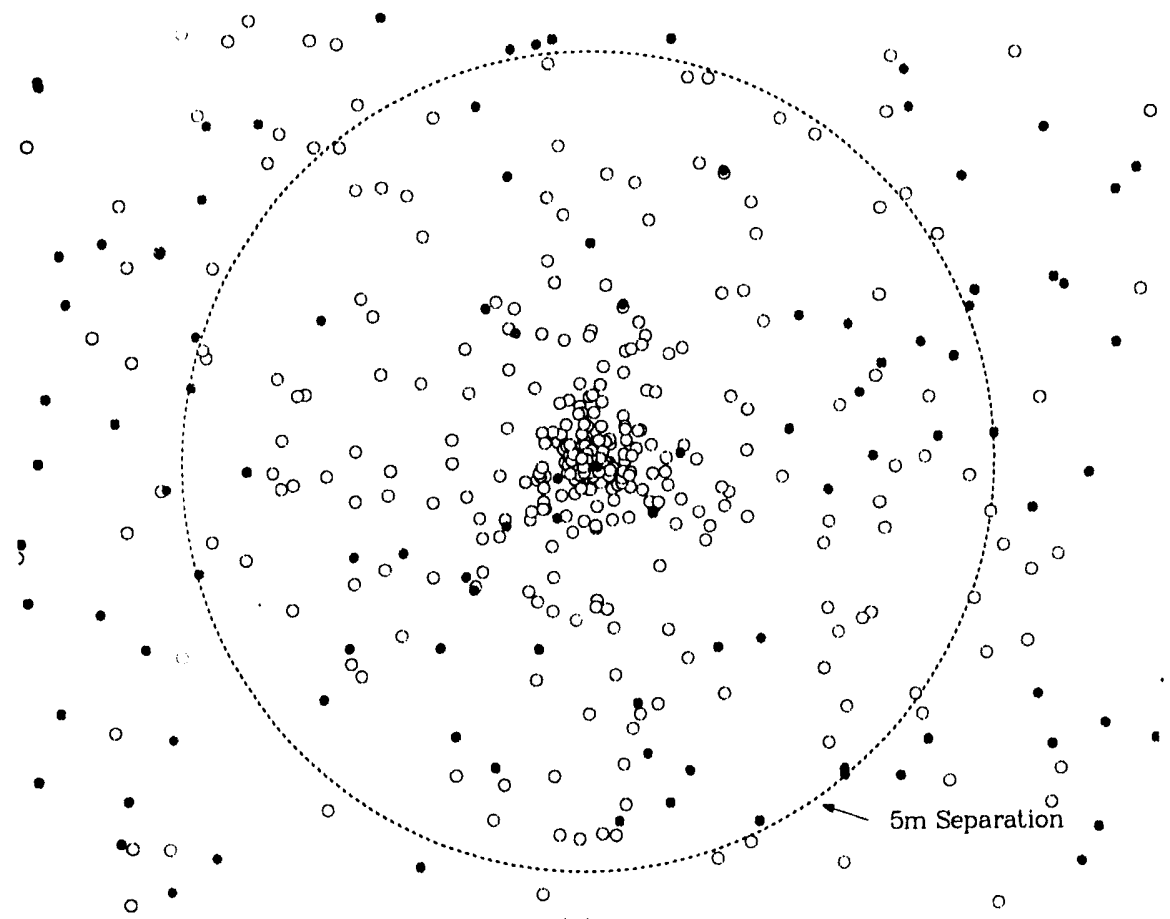

(a)

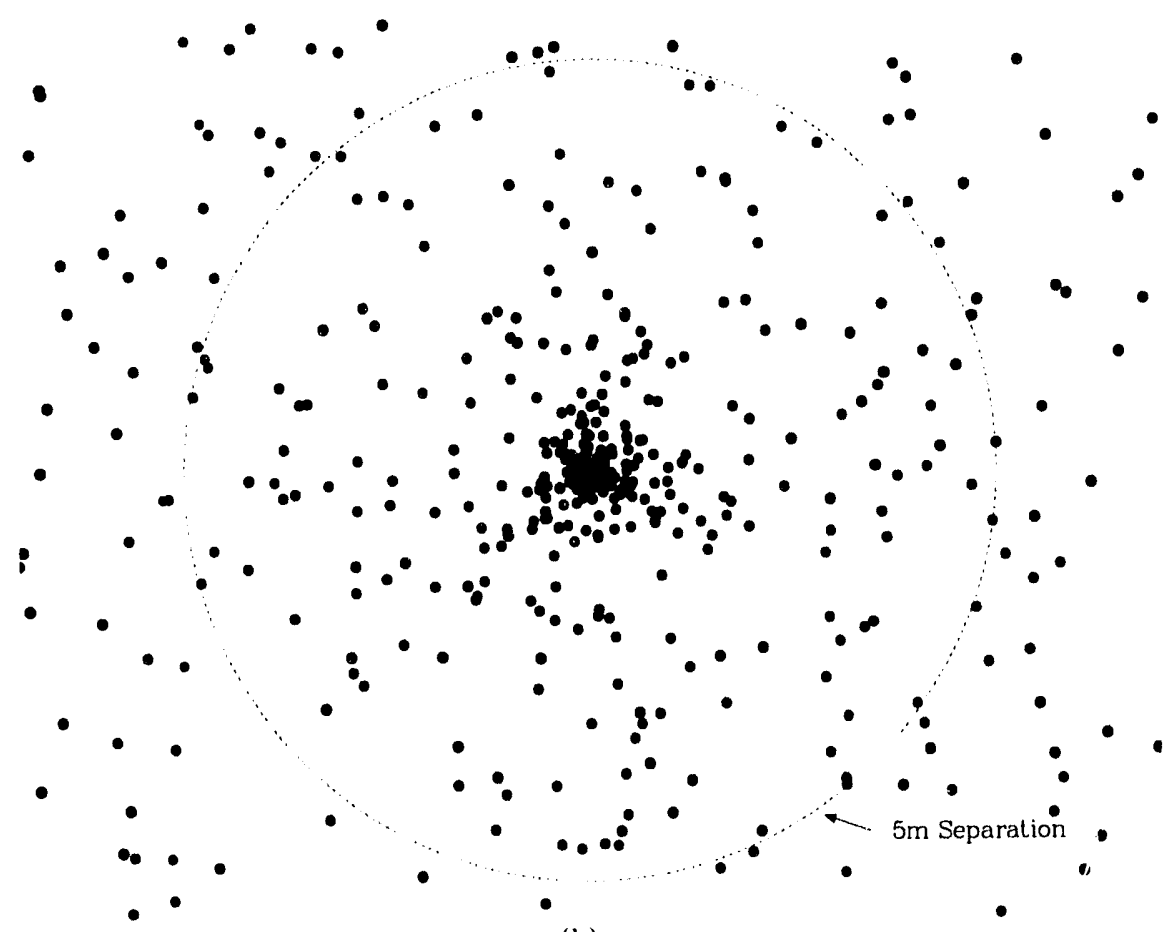

(b)

Fig. 3. 1 a,b. Simulation of detector counting in a radiation field produced by a $1 / r^{2}$ source flux and a uniform background. In the upper panel (part a), the open circles are source counts; the closed circles are background counts. In the lower panel (part b), no distinction is made between source and background counts. 
detecting a significant change in count rate unless the source is near at hand. This difficulty is further emphasized by Fig. 3.1b, which drops the artificial distinction between counts from the source and the background. Only at very close approaches is the variation in count rate clearly observable.

Directional Radiation Fields. Instead of focusing solely on variations in count rate, Fig. 3.2a uses the same simulation to illustrate the concept of a directional radiation field. The solid line segments radiate from the source location; the dotted segments have randomly chosen directions. Again, the $S / B$ ratio of 5:1 at the 5-m separation is apparent. In this case, however, the combination of source and background also affects the average direction of the local flux, not just the variation in count rate from point to point. This assertion is supported by Fig. $\mathbf{3 . 2}$ b, which again drops the distinction between counts from the source and background. For measurements far from the source, the directions of the detected particles are random; as the source is approached, the fraction of particles with a definite orientation increases to $100 \%$. In terms of polarization observables, one refers to the radiation fields for near and far sources as having polarization magnitudes that approach 1.0 and 0.0 , respectively. This interpretation leads to two conclusions: (1) The net direction of the local polarization vector indicates the most likely direction for the radiation source, and (2) the magnitude $P$ of the polarization increases from 0.0 to 1.0 as the source is approached. Thus, a nonzero polarization measurement may provide a sensitive indicator of a directional radiation field - and hence a localized neutron source.

Basic Polarization Observables. Typically, however, the polarization of a radiation field cannot be observed directly, but it must be deduced from differences between the count rates in a mul vement detection system. In our case, we used the four-rod instrument of Fig. 2.1 with its long axis vertical, so that each rod faced a different direction. In the formalism of polarization observables, the sensitivity of a detector to the polarization $P$ of a radiation field is referred to as its analyzing power $A$, which varies from 0.0 (no response) to 1.0 (perfect response). In this frameworl, a measurement's polarization characteristics depend on both the field and the detector, which leads to a product referred to as the asymmetry $R=P A$. It is this count-rate asymmetry that forms the central polarization quantity obtained in the measurements.

Summary. This section has provided a simple illustration of the effect on search behavior of background contributions, of the inverse-square falloff in signal flux, and of counting statistics. We have used the radial characteristics of the emissions from a point source to introduce the concepts of a polarized radiation field and a directional radiation detector. This approach has resulted in an application of detector directionality not anticipated in the idealized simulations of Ref. 1. For sources at moderate separations (up to a few meters), most of the neutron flux is incidrnt on the detector from a particular direction, which produces a definite difference between the count rates for the different elements. At larger separations, however, thr direct source contribution decreases as $1 / r^{2}$, and most of the detected neutrons have scattered from the air, walls, or floor. Thus, the polarization of the radiation field provides a measurement of the signal-to-background ratio and hence an estimate of the nearness of the radiation source. The central result is the equation $R=P A$, which connects the field and detector parameters $P$ and $A$ to a measurable count-rate asymmetry $R$. We have also arrived at two significant predictions that can be tested against experimental results: (1) the polarization direction indicates the source angle, and (2) the polarization magnitudr depends on the signal-to-background characteristics of the radiation ficld. 


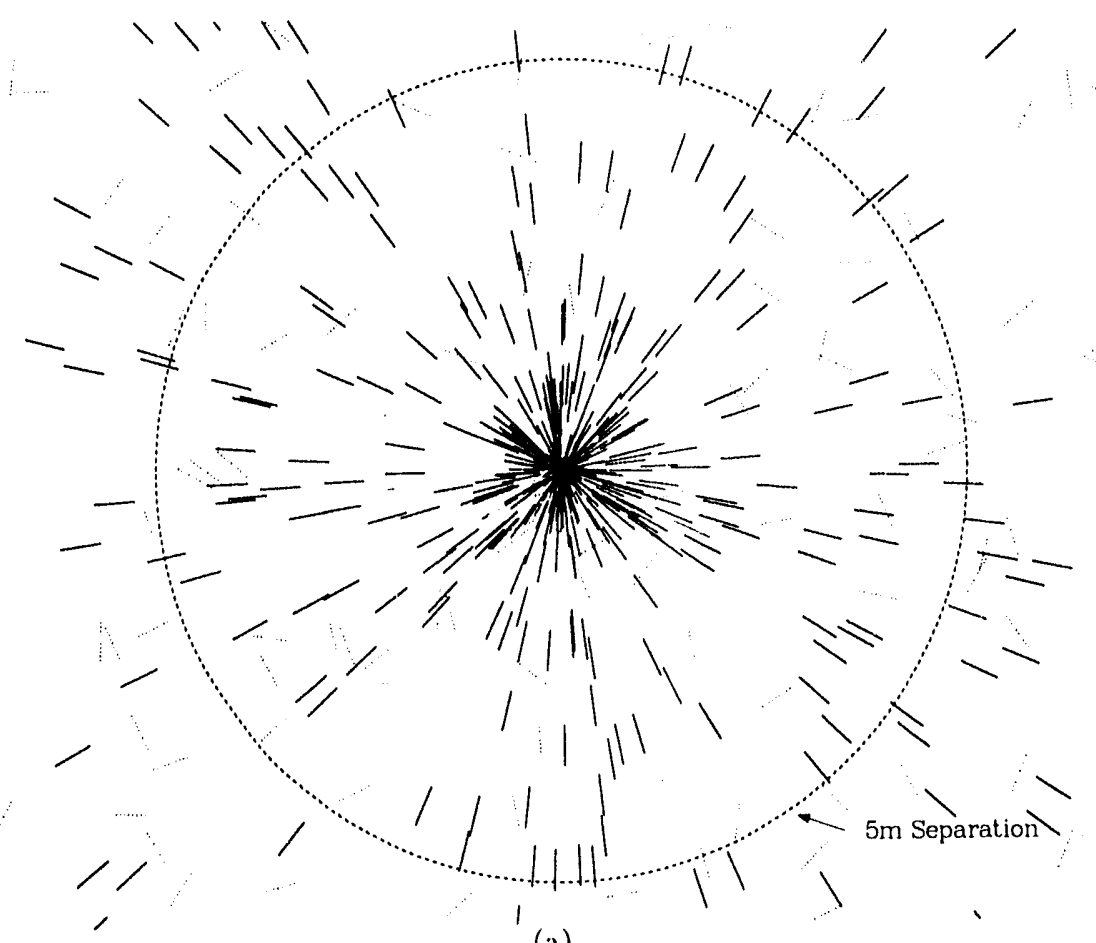

(a)

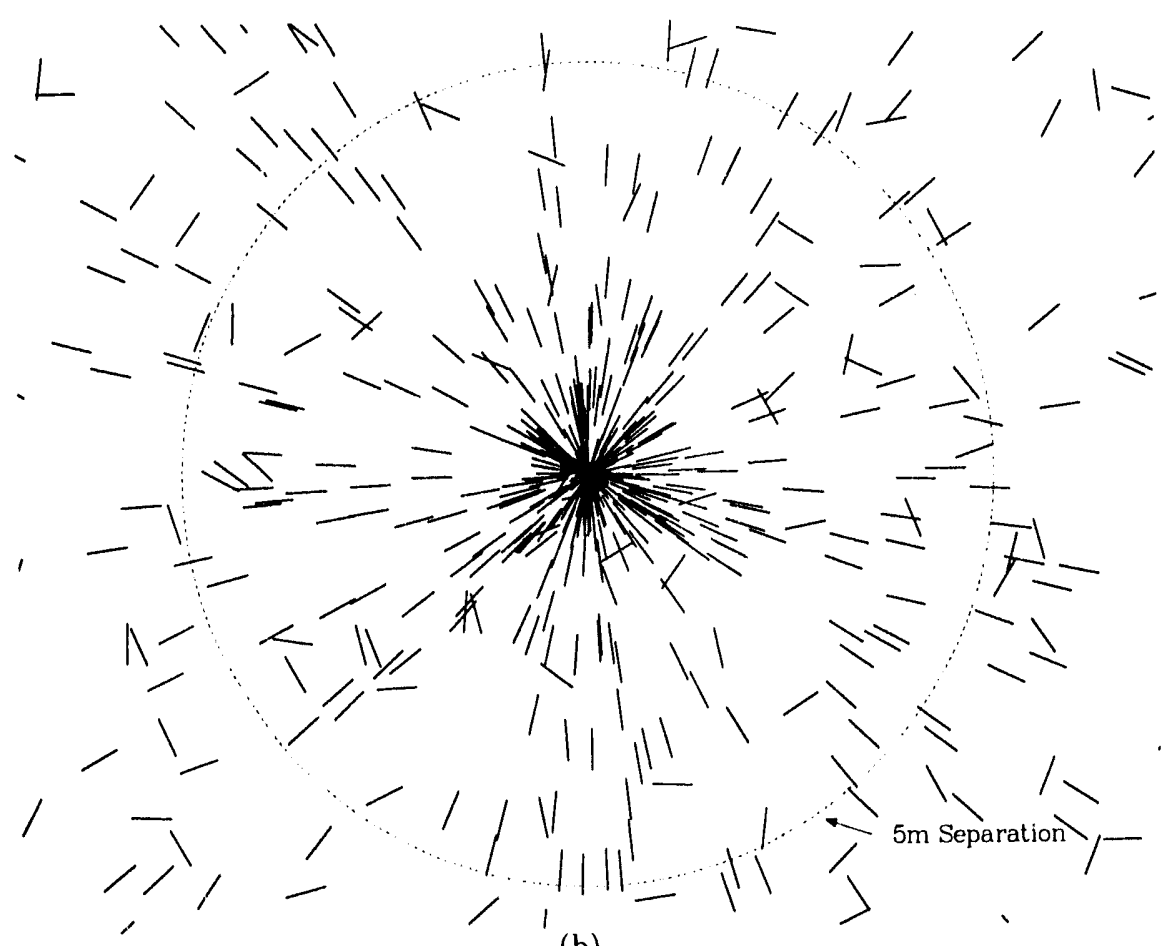

(b)

Fig. 3.2a,b. Simulation of a directional radiation field produced by a $1 / r^{2}$ source flux and a uniform background. In the upper panel (part a), the solid lines indicate the directions of source particles; the dotted lines indicate a random background. In the lower panel (part b), no distinction is made between source and background particles. 


\section{POLARIZATION MEASUREMENTS}

Overview. This section begins by relating the conceptual four-element polarization detector of the previous section to the actual instrument used in the measurements. After developing a mathematical formalism based on standard polarization analyses, we use examples from the measurements to test the predictions made in the previous section concerning the direction and magnitude of the measured polarization vector. Most of the measurements were made for distributed sources located at various distances in front of the detector, all at about the same angle. The calculated direction therefore serves mainly as a consistency check, although the magnitude of the polarization should decrease as the source separation increases.

Experimental Configuration. The measurements were made with the sources placed roughly in front of the detector at recorded separations of $54-1160 \mathrm{~cm}$ (center to center). To reduce the count rate from gamma rays, the detector was surrounded by at least $0.95 \mathrm{~cm}$ of lead sheet on all sides. Figure 4.1 connects our corceptual polarization analyzer with the physical four-rod instrument shown in Fig. 2.1, showing the numbering of the four rods, the angle coordinates, and the right/left/front/back directions. The attenuation through the detector and differences in solid angle produce a unique pattern of count-rate ratios. At the source position shown, Rod 2 would have the highest rate, followed by Rods 1,4 , and 3 , respectively. As the source angle $\theta$ increases toward $45^{\circ}$, the count-rate ratio between Rods 1 and 4 decreases to 1:1, while that between Rods 1 and 3 increases to its maximum value, which is about $4: 1$ for the geometry and materials of our particular detector.

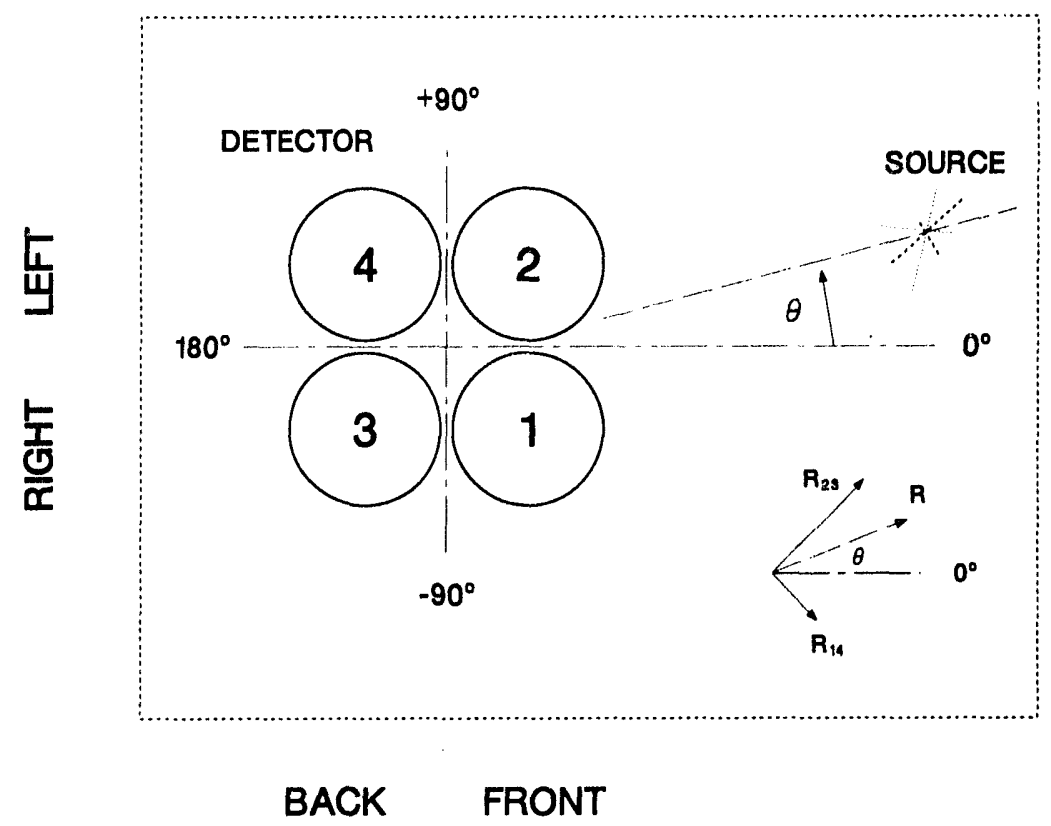

Fig. 4.1. Detector and source geometries, with angle coordinates shown from above. Rods 1 and 2 are on the front side of the detector and face the source; Rods 3 and 4 are on the rear side. A source angle of zero degrees is straight ahead. The inset shows a detector-centered coordinate system used in the asymmetry calculations. 
Directional Algebra. The formal directional calculations are surprisingly simple. Three observables are obtained: (1) the angle-averaged neutron flux at the detector location, (2) the magnitude of the local polarization, and (3) the direction of the local polarization. Alternatively, this information can be viewed as a scaler neutron flux and a vector neutron polarization, which correspond to the single-rod count rate and the multirod count-rate asymmetry. In Ref. 1, the calculations used differences between the count rates for opposite rods; the current software emphasizes the ratios between these count rates. The local average flux $\Phi$ and its fractional error $\epsilon_{\Phi}=l \Phi / \Phi$ are obtained from the four individual-rod yields and errors $\phi_{i} \pm d_{i}$ as

$$
\begin{gathered}
\Phi=\left(\phi_{1}+\phi_{2}+\phi_{3}+\phi_{4}\right) / 4 \\
\epsilon_{\Phi}=\left(d \phi_{1}^{2}+d \phi_{2}^{2}+d \phi_{3}^{2}+d \phi_{4}^{2}\right)^{\frac{1}{2}} / 4 \Phi .
\end{gathered}
$$

The measurable directional parameter is the asymmetry $R$ and fractional error $\epsilon_{R}$. For simplicity, the detector axes are oriented diagonally through the 1-4 and 2-3 detector rods, and the source angle $\theta$ is shifted by $\pi / 4\left(45^{\circ}\right)$ to align the axis with zero degrees for sources directly in front of Rods 1 and 2 (see Fig. 4.1). Our approach treats the count-rate ratios as the components $\left(R_{14}, R_{23}\right)$ of a vector whose length and direction are $R$ and $\theta$. The calculations proceed as follows:

$$
\begin{gathered}
\alpha=\phi_{1} / \phi_{4}, \quad \beta=\phi_{2} / \phi_{3} \\
R_{14}=(\alpha-1) /(\alpha+1), \quad R_{23}=(\beta-1) /(\beta+1) \\
R^{2}=\left(R_{14}^{2}+R_{23}^{2}\right) \\
u=R_{23} / R_{14}, \quad \theta=\tan ^{-1} u-\pi / 4 .
\end{gathered}
$$

The errors are based on the fractional errors $\epsilon_{i}=d \phi_{i} / \phi_{i}$ for the four rods and the corresponding $\epsilon_{14}$ and $\epsilon_{23}$ values for the two components $\left(R_{14}, R_{23}\right)$ :

$$
\begin{gathered}
d \alpha=\alpha\left(\epsilon_{1}^{2}+\epsilon_{4}^{2}\right)^{1 / 2}, \quad d \beta=\beta\left(\epsilon_{2}^{2}+\epsilon_{3}^{2}\right)^{1 / 2} \\
d R_{14}=2 /(\alpha+1)^{2} d \alpha, \quad d R_{23}=2 /(\beta+1)^{2} d \beta \\
\epsilon_{R}=\left(R_{14}^{2} d R_{14}^{2}+R_{23}^{2} d R_{23}^{2}\right)^{1 / 2} / R \\
d u=u\left(\epsilon_{14}^{2}+\epsilon_{23}^{2}\right)^{1 / 2} \\
d \theta=d u /\left(1+u^{2}\right) .
\end{gathered}
$$

In this arrangement, $\Phi$ measures the average neutron intensity, $\theta$ gives the source direction, and $R$ indicates the asymmetry across the detector-three basic parameters.

Detector Analyzing Power. To use the measured asymmetries $R$ to obtain the field polarizations $P$, we must obtain an estimated value for the detector analyzing power $A$. Because the asymmetries measured for nearby sources are mainly caused by attenuation across the detector, they should have essentially constant values that are characteristic of the detector material and geometry, not the signal-to-background characteristics of the radiation field. The Monte Carlo calculations in Ref. 1 did not include background effects, so they should provide a good estimate of the detector's intrinsic $A$-value. The calculated asymmetries have a maximum value near 0.66 for angles through the rods $\left( \pm 45^{\circ}, \pm 135^{\circ}\right)$, a minimum value near 0.57 at the intermediate angles $\left( \pm 22.5^{\circ}, \pm 37.5^{\circ}\right.$, etc. $)$, and a value near 0.62 at the gaps between each pair of rods $\left(0^{\circ}, \pm 90^{\circ}, 180^{\circ}\right.$, that is, our experimental situation). To check these calculations, we can use a calibration measurement made with a ${ }^{252} \mathrm{Cf}$ fission source placed in front of the detector at a distance of $54 \mathrm{~cm}$ (Run $802 \mathrm{in}$ the experiment logs). This run has an asymmetry value $R=0.694 \pm 0.6 \%$. Furthermore, 
the weighted average of the $R$-val:: ss for all the measurements is $R=0.67$ with a standard deviation of 0.21 . Thus, the measured asymmetries of $0.6-0.7$ for most of the distances and angles inclucled in our data set are consistent with an interpretation in terms of attenuation in the detector material. Because all the present measurements were made at a nominal source angle of zero degrees, we will use the calculated value $R=0.62$ as our standard detector analyzing power $A$. With this assumption, the measured asymmetries can be used to calculate the polarization magnitude as

$$
P=R / A, \quad \epsilon_{P}=\epsilon_{R} / A .
$$

For some applications, $\mathrm{i}^{+}$is also useful to fold the intensity and polarization into a single quantity, the flux gradient $G$ across the detector, which is given as

$$
\begin{gathered}
G=P \Phi \\
\epsilon_{G}^{2}=\epsilon_{P}^{2}+\epsilon_{\Phi}^{2} .
\end{gathered}
$$

This quantity estimates the portion of the flux that is associated with the radiation source, as opposed to that for the local background.

Sample Calculations. We can use the values from the ${ }^{252} \mathrm{Cf}$ calibration run to illustrate the behavior of the directional algebra. (Table I collects the relevant analysis results from all runs discussed in this report.) For the calibration run, the four single-rod fluxes (integrated from $0.75 \cdots 10.0 \mathrm{MeV}$ ) are

$$
\begin{aligned}
& \phi_{1}=0.1741 \pm 0.7 \% \mathrm{n} / \mathrm{cm}^{2} / \mathrm{s} \\
& \phi_{2}=0.2221 \pm 0.7 \% \mathrm{n} / \mathrm{cm}^{2} / \mathrm{s} \\
& \phi_{3}=0.0574 \pm 1.7 \% \mathrm{n} / \mathrm{cm}^{2} / \mathrm{s} \\
& \phi_{4}=0.0808 \pm 1.3 \% \mathrm{n} / \mathrm{cm}^{2} / \mathrm{s} .
\end{aligned}
$$

There is a clear difference between the front $\left(\phi_{1}, \phi_{2}\right)$ and rear $\left(\phi_{3}, \phi_{4}\right)$ count rates. The average flux (converted from units of $\mathrm{cm}^{2}$ to $\mathrm{m}^{2}$ for convenience) is $\Phi=1336 \pm 0.5 \% \mathrm{n} / \mathrm{m}^{2} / \mathrm{s}$, the polarization magnitude is $1.12 \pm 0.01$, the polarization angle is $\theta=13.1 \pm 0.5^{\circ}$, and the flux gradient is $G=1495 \pm 0.9 \% \mathrm{n} / \mathrm{m}^{2} / \mathrm{s}$. The polarization value is larger than 1.0 because solid-angle effects at short separations result in an unusually large detector analyzing power. Thus, our $A$-value of 0.62 is appropriate only for sources at separations large compared with the detector size, that is, beyond about 1-2 $\mathrm{m}$. The source angle of $13.1^{\circ}$ for the calibration run is also anomalous. For a source located at $54 \mathrm{~cm}$, this angle corresponds to a displacement of $12.6 \mathrm{~cm}$ to the right; unfortunately, no precise right/left source position was recorded for this run. A different ${ }^{252} \mathrm{Cf}$ measurement (Run 245 in the logs), probably made with the source immediately in front of the detector, gives a more reasonable angle of $-1.3 \pm 0.3^{\circ}$. In fact, the angle of $13.1^{\circ}$ is one of the largest measured in the entire set of runs, with one apparent exception. $\Lambda$ later measurement (Run 817 in the logs) with another source gives the result

$$
\begin{aligned}
\phi_{1} & =0.1743 \pm 4.6 \% \mathrm{n} / \mathrm{cm}^{2} / \mathrm{s} \\
\phi_{2} & =0.0860 \pm 6.8 \% \mathrm{n} / \mathrm{cm}^{2} / \mathrm{s} \\
\phi_{3} & =0.1694 \pm 5.5 \% \mathrm{n} / \mathrm{cm}^{2} / \mathrm{s} \\
\phi_{4} & =0.0635 \pm 11.5 \% \mathrm{n} / \mathrm{cm}^{2} / \mathrm{s} .
\end{aligned}
$$


Here, the fluxes for Rods 1 and 3 are higher than those for Rods 2 and 4 (not Rods 1 and 2 vs Rods 3 and 4 ). The calculated angle of $-80.1 \pm 3.7^{\circ}$ implies that the detector was accidentally rotated by $+90^{\circ}$ See Fig. 4.1 ), which gives a true source angle of $9.9 \pm 3.7^{\circ}$ ! (The values listed for this run in Table I include the assumed $+90^{\circ}$ correction.) Ironically, this fortuitous rotation provides one of the best confirmations in the present experiment fc: the detector's directional capability.

\section{TABLE I. Analysis Results}

The notation in the table is as follows:

\section{On-line Quantities}

J Index (1-22) for each set of runs. Similar runs are grouped together.

RUN First run in the original set of run numbers. For example, Run 802 is Runs 82-83; Run 817 is the sum of Runs $817-819$.

NAME Mnemonic for the particular source, usually taken from the run logs.

VIEW Viewing angle (deg) for the source, from the run logs.

DIST Separation $(\mathrm{cm})$ between source and detector, from the run logs.

TIME Measurement time interval $(\mathrm{min})$, calculated from the data by converting the number of 96-s event-data frames into minutes.

\section{Directional Quantities}

ANGL Source angle and error in degrees, obtained fron the asymmetry analysis.

AFLX Average flux $\Phi$ at the detector, given in neutrons $/ \mathrm{m}^{2} / \mathrm{s}$ and averaged over all four rods. $\epsilon_{\Phi}$ is the percentage error.

TFLX Front-rod flux integral $\phi_{f}$ at the detector. $\epsilon_{f}$ is the percentage error.

SFLX Average background-subtracted flux integral $\phi_{a} . \epsilon_{a}$ is a percentage error.

BFLX Background correction $\left(\phi_{f}-\phi_{a}\right)$.

POLN Polarization $P$ measured by the detector.

GFLX Flux gradient $G=P \Phi$, with percentage error $\epsilon_{i}$.

S/T Signal-to-total ratio SFLX/TFLX.

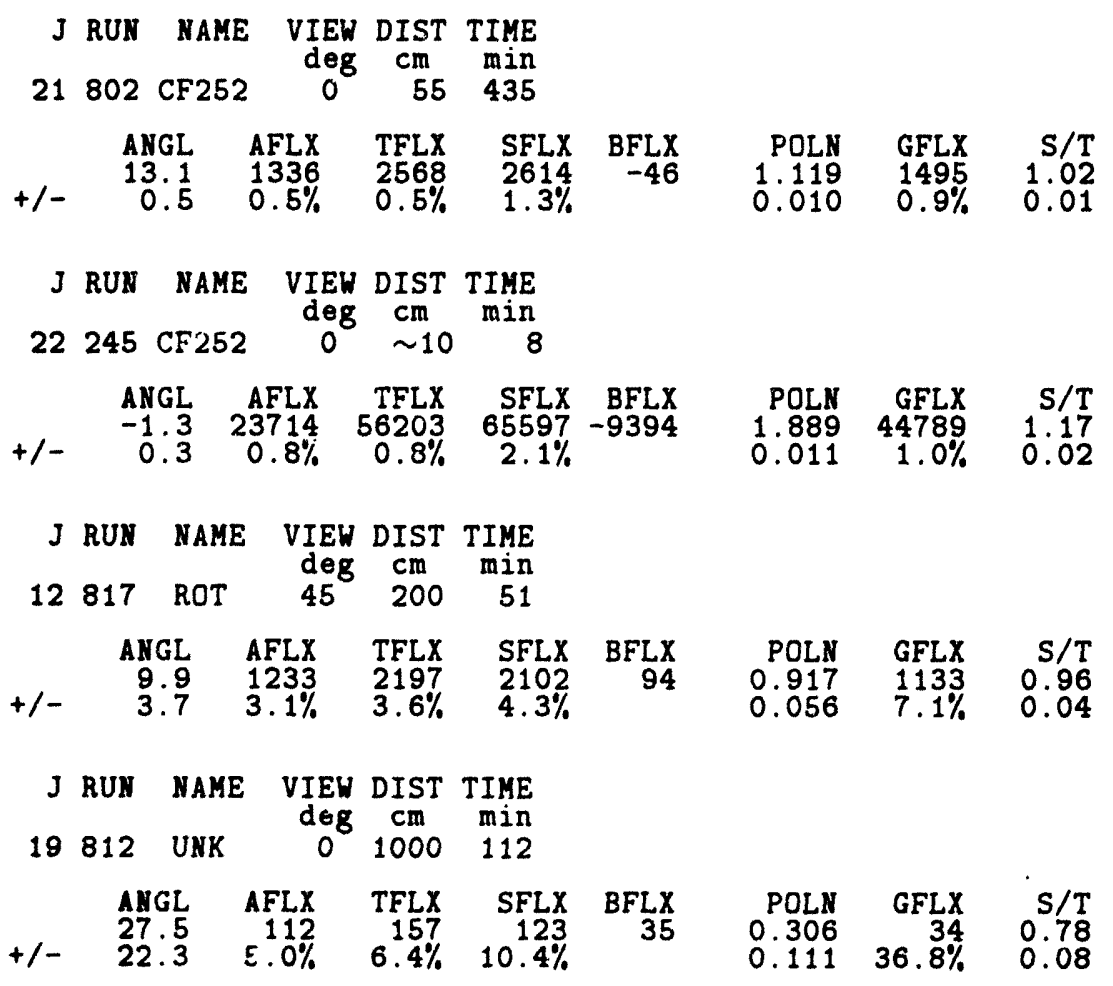


Angle Accuracies. The discussion in Sec. 3 of the polarized radiation fields resulted in two predictions that can be tested against the measurements. The first prediction was that the observed polarization direction should correspond to the angle of the source location. In Ref. 1, Monte Carlo calculations were made for two different source/detector geometries, one corresponding to incident parallel beams (distant sources) and the other to point (nearby) radiation sources. These calculations produced standard deviations of $4.5^{\circ}$ and $5.9^{\circ}$, respectively, from the true values. Turning to the present measurements, the experiment was unfortunately not designed to test the accuracy of the angle estimates, so all sources were nominally placed at zero degrees. With the significant exception of the run with the rotated detector assembly, the data are a meaningful test only to the extent that the angle values are all consistent with zero. In this view, the present ixperiment with large, distributed sources at a single angle complements the point-source measurements described previously in Ref. 1. For our distributed sources, that is, omitting the two ${ }^{252} \mathrm{Cf}$ point-source calibrations, the unweighted average angle is $4.8 \pm 1.8^{\circ}$. For the point sources of Ref. 1, measurements are available at source angles of $45^{\circ}$ and $90^{\circ}$. Using the present software, the corresponding measured angles are $41 \pm 5^{\circ}$ and $88 \pm 1^{\circ}$, in goou agreement. Based on these combined sets of measurements and simulations, it is reasonable to expect angle accuracies within $\sim 5^{\circ}$ for sources located near the eight symmetry planes $\left(\right.$ at $0^{\circ}$, $\pm 90^{\circ}, 180^{\circ}, \pm 45^{\circ}$, and $\pm 135^{\circ}$ ). As discussed in Ref. 1, accuracies at other angles may be somewhat worse because of multiple-scattering and solid-angle effects. However, the expected accuracies should be completely adequate for most practical applications.

Polarization versus Separation. The second prediction of our conceptual polarization model concerned the relationship between the magnitude of the polarization vector and the signal-to-background ratio for the local radiation field, which should both decrease as the source/detector separation increases. To test this prediction, Fig. 4.2 shows the correlation between the source separation and the measured polarization. Most of the measurements for nearby sources are consistent within errors with unit polarization, as expected from our definition of the analyzing power $A$. An exception is the two calibration points (solid symbols), which were obtained with small sources placed near the detector (at $50-150 \mathrm{~cm}$ ). The solid circle is the ${ }^{252}$ (f measurement; the solid square uses a nonweapon ${ }^{240} \mathrm{Pu}$ source. These slightly high $P$-values are assumed to be a solid-angle effect associated with the very short sonrce/detector separation. The open symbols show measurements made with various distributed sources. Similar symbols are used for related objects, and the thin lines connect measurements made with the same object but at different separations. The first encouraging result is the five points at large separations $(1000-1200 \mathrm{~cm})$, whose measured $P$-values of 0.40 .6 are consistent with the value $P=0.5$ expected for the equal fluxes of signal and background at a separation of $10 \mathrm{~m}$ (see Sec. 3). The second result is the set of three solid lines, each for the same source at different separations, which have polarizations that clearly decrease as the source separation increases. On the other hand, there remain two anomalous sets of runs that must addressed in a subsequent report on the individual masurements. First, the three points connected by the dashed line seem to have the wrong separation dependence. but in this case the recorded separations are not center-to-center distances but aro measured extrrnally to a large container. Second, there are three smill scluares at $200 \mathrm{~cm}$ with large uncertainties but polarizations that nevertheless appear to be somewhat too high. Overall, however, the clear evidence is that polarizations near $I^{\prime}=1.0(R \sim 0.62)$ correspond to nearby sources, while $P$-values below $0.8(R<0.5)$ indicate sources at large separations.

Search Applications. A realistic example of the utility of the directional information is provided by a measurement with an "unknown" source (Run 812 in the logs). (For reference, this meanurement is indicated by a superimposed square-and-triangle symbol in Fig. 4.2.) In this rase, a sonres at a separation of $1000 \mathrm{~cm}$ produced an average flux 


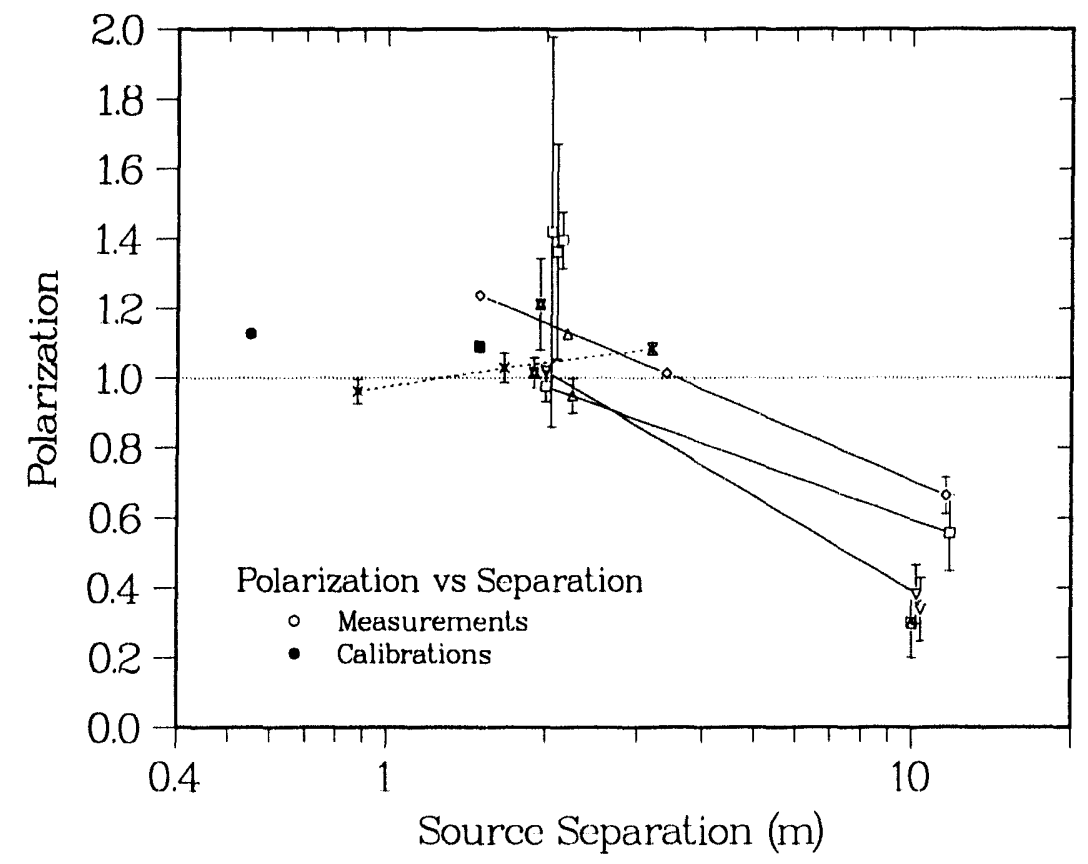

Fig. 4.2. Correlation between source separation and measured polarization $P$. The slanted lines connect measurements made with the same sources but at different separations.

at the detector (see Table I) of 112 neutrons $/ \mathrm{m}^{2} / \mathrm{s}$, which is only a few times the highly variable background rate from neutrons generated by cosmic rays. ${ }^{5}$ Because of this large nonstatistical uncertainty, it would be difficult to assert the existence of a radioactive source, much less its likely location, even with the $5 \%$ statistical error of a 112 -min measurement. However, few mechanisms exist to produce polarized natural backgrounds, so the measured polarization of $0.31 \pm 0.11$ is much more significantly nonzero, despite its larger statistical error. Furthermore, in a search scenario the estimated direction of $28 \pm 22^{\circ}$ provides a reasonable heading for additional measurements. This possibility will be explored further in the next section.

Discussion Given the complexity of effects such as source and detector solid angles, neutron attenuation, and scattering between adjacent detector segments, the proposed polarization algebia may seem somewhat simplistic. From our previous report, ${ }^{1}$ we expected that the most important directional information provided by a segmented detector would be the angle of the incident neutron flux. The interpretations and analyses in this section and the previous one indicate that source direction is only part of the larger concept of a polarized radiation field, which carries both direction and magnitude information. As demonstrated in this section, the polarization direction indicates the source angle, and the magnitude indicates the signal-to-background ratio, which provides an estimate of the nearness of the radiation source.

\section{SEARCH SIMULATIONS}

Overview. The previous sections proposed the concept of a polarized radiation field and presented data to support the existence of both the field and a simple detector with 
suitable response. This section addresses the usefulness of the concept for improving the performance of searches for radioactive sources. Our evaluation is based on Monte Carlo simulations that include four important features: (1) reasonable models of the source, background, detector properties, and counting statistics, (2) standard search parameters such as integration times, step sizes, and success criteria, (3) detectors with various capabilities for count-rate or directional information, and (4) search strategies that specify how the information is to be used. Although our simulations are intended to be realistic, we stress that the emphasis will be on the relative performance of the different approaches, not on the absolute source separations or success rates.

Search Models and Parameters. Several assumptions are common across the different simulations. All use step sizes of $4 \mathrm{~m}$ and integration times of $60 \mathrm{~s}$. All use the actual detector's frontal area of $300 \mathrm{~cm}^{2}$, average detection efficiency of 0.05 , and analyzing power of 0.62 . The source emission rate is $10^{5}$ neutrons/s, and the uniform background count rate is 2 counts/s. This background rate, which is more than ten times higher than our maximum measured rate, has been increased because our subtraction of random coincidence rates contributes a statistical uncertainty whose precise effect is difficult to estimate. Because much of our emphasis will be on differences between the directional and nondirectional strategies, it is important to point out that our simulated four-rod directional detector is assumed to have the same volume as the equivalent single-element instrument, that is, the single-rod detector has four times the count rate of each element of the multirod detector. Another critical assumption concerns the simple relation between the polarization of the radiation field and its $S / B$ value: $P=S /(S+B)$ Finally, in all cases the statistical uncertainties are given by square root of the total number of detected counts, $T=S+B$.

Information and Strategies. In distinguishing among the different cases, we assume that the detector can provide the types of information described in our discussion of polarization algebra: the average flux $\Phi$, the polarization magnitude $P$, the flux gradient $G=P \Phi$, the two polarization components $\left(P_{x}, P_{y}\right)$, and the polarization direction $\theta$. For the latter two directional searches, referred to as $P_{x y}$ and $\theta$, each individual measurement provides a heading for a new search. Specifically, for a $\theta$ search, each 60 -s measurement is followed by a $4-\mathrm{m}$ step in exactly the direction given by the estimated source angle. For a $P_{x y}$ search, in the detector coordinates the $x$ and $y$ components are essentially the front/back $(x)$ and right/left $(y)$ asymmetries. Thus, a positive $P_{x}$ value results in another step in the same (forward) direction; a negative $P_{x}$ value reverses direction; two successive negative $P_{x}$ measurements cause a step to the left or right, depending on the sign of $P_{y}$. The other approaches are similar to the $P_{x y}$ strategy, but they depend on a change (a $\Delta$ ) between successive measurements. In such $\Delta$ searches, whether using $\Phi, P$, or $G$, a positive $\Delta$ causes a step in the same direction; a negative $\Delta$ reverses the direction; two negative $\Delta$ 's cause a step $9\left(0^{\circ}\right.$ to the right. Finally, for comparison an additional calculation was run using a "no-detector" strategy $N$, in which each step is taken in a random direction. Note that only the $\theta$ and $N$ searches can move diagonally; all others are constrained to a rectilinear search grid with an arbitrary angular orientation.

Search Durations, Simple Case. To introduce the search simulations in the simplest possible context, our first set of runs neglects the effect of counting statistics. (As such, the no-detector $N$ case is omitted.) Figure 5.1 shows that the results fall into three classes that depend only on the consequences of the search's diagonal or rectilinear grid strategies. The angle search ( $(\theta)$ reaches the source in the minimum time; for example, using 4-m steps to approach within $8 \mathrm{~m}$ of a source at $55 \mathrm{~m}$ requires $(55-8) / 4=12$ steps or 13 separate 1-min measurements. Because the $\Delta$ strategies depend on the results of two measurements and must move along a rectilinear grid, they result in longer searches. However, without statistical uncertajuties, all are based on equivalent "perfect" information and all obtain 


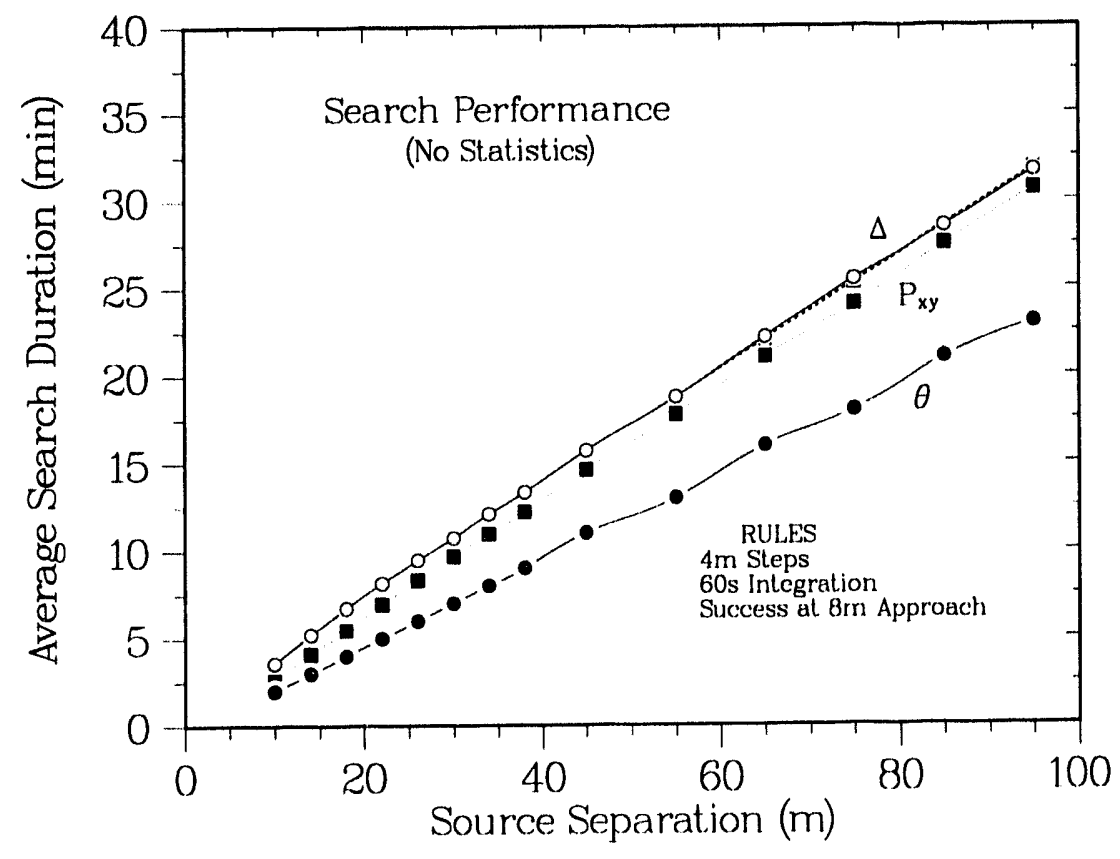

Fig. 5.1. Average durations for searches without statistical uncertainties. The various strategies collapse into only three different cases.

the same result. The $P_{x y}$ strategy is an intermediate case; it moves on a rectilinear grid, but it requires only a single measurement and always turns to the left or right correctly.

Success Rates, Simple Case. Only for the $\theta$ search does the detector always reach the source in exactly the minimum number of steps; all the rectilinear cases obtain a range of durations that depend on the particular starting angle (which is chosen randomly). Some starting angles happen to achieve the minimum value of a $\theta$ search, but most take far longer, and the spread between the shortest and longest durations increases as the separation increases. This range of values can be expressed in terms of the search success rate as a function of search duration, as shown in Figs. 5.2a,b for source separations of $10 \mathrm{~m}$ and $34 \mathrm{~m}$. For example, if a success rate of at least $80 \%$ is specified, at a $10-\mathrm{m}$ separation the $\theta, P_{x y}$, and $\Delta$ approaches require durations of at least $2 \mathrm{~min}, 3 \mathrm{~min}$, and $5 \mathrm{~min}$, respectively. The corresponding values at an $18 \mathrm{-m}$ separation (not shown) are $4 \mathrm{~min}$, $5 \mathrm{~min}$, and $7 \mathrm{~min}$; the $34-\mathrm{m}$ values are $8 \mathrm{~min}, 12 \mathrm{~min}$, and $13 \mathrm{~min}$. As suggested by the slopes in Fig. 5.1, the differences remain about the same as the separation increases.

Search Durations, with Statistics. As should be expected, including the effect of counting statistics profoundly increases the complexity of the calculations. Most importantly, it now becomes possible for individual searches to fall into a "random walk" that has a negligible chance of finding the source. 'To avoid this possibility, an additional rule is invoked that returns the detector to its original location if its net displacement reaches 1.5 times the source separation. The search is then continued, but starting in another random direction. The search durations from these simulations are illustrated in Fig. 5.3a. There has been a dramatic increase in the time required for most cases, and there is now a clear ordering to the major strategies: $\theta, P_{x y}, \Phi, G, P$, and $N$. Note that the time dependence for the random, no-detector $(N)$ search is almost a straight diagonal line. On our double-logarithmic plot with three decades for time and one decade for separation, this 


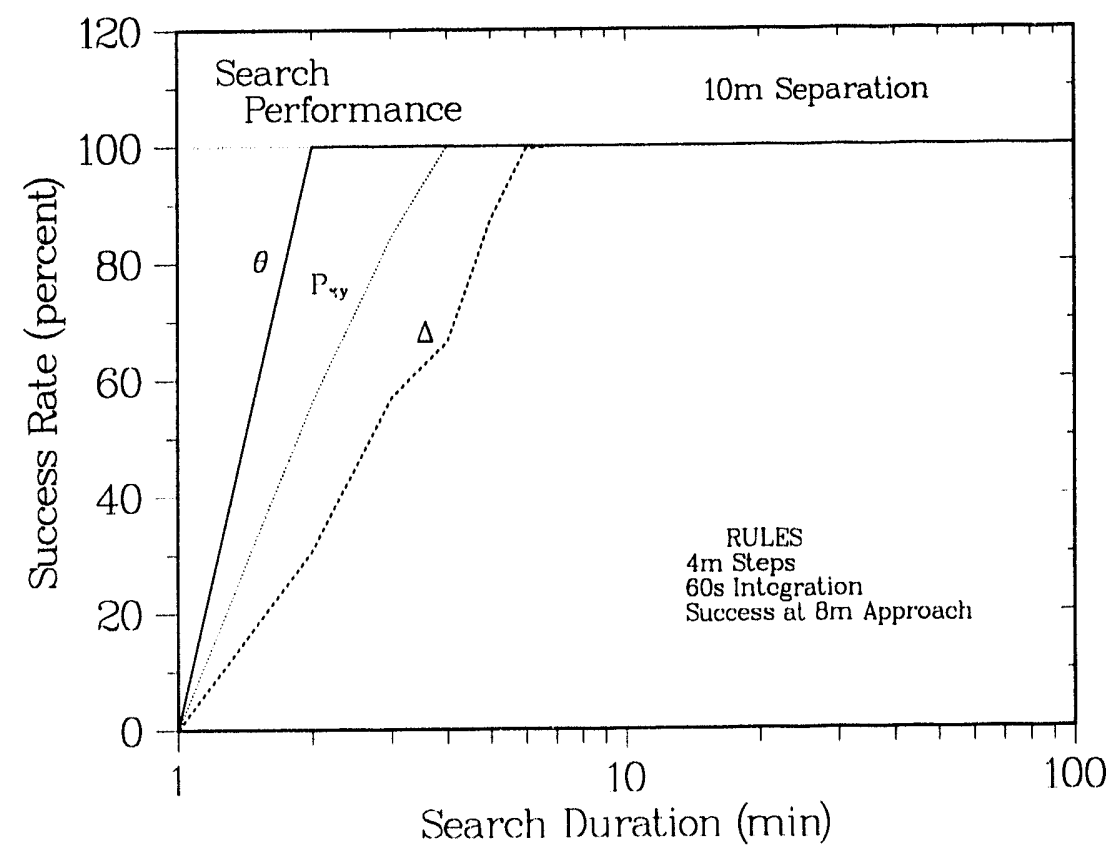

(a)

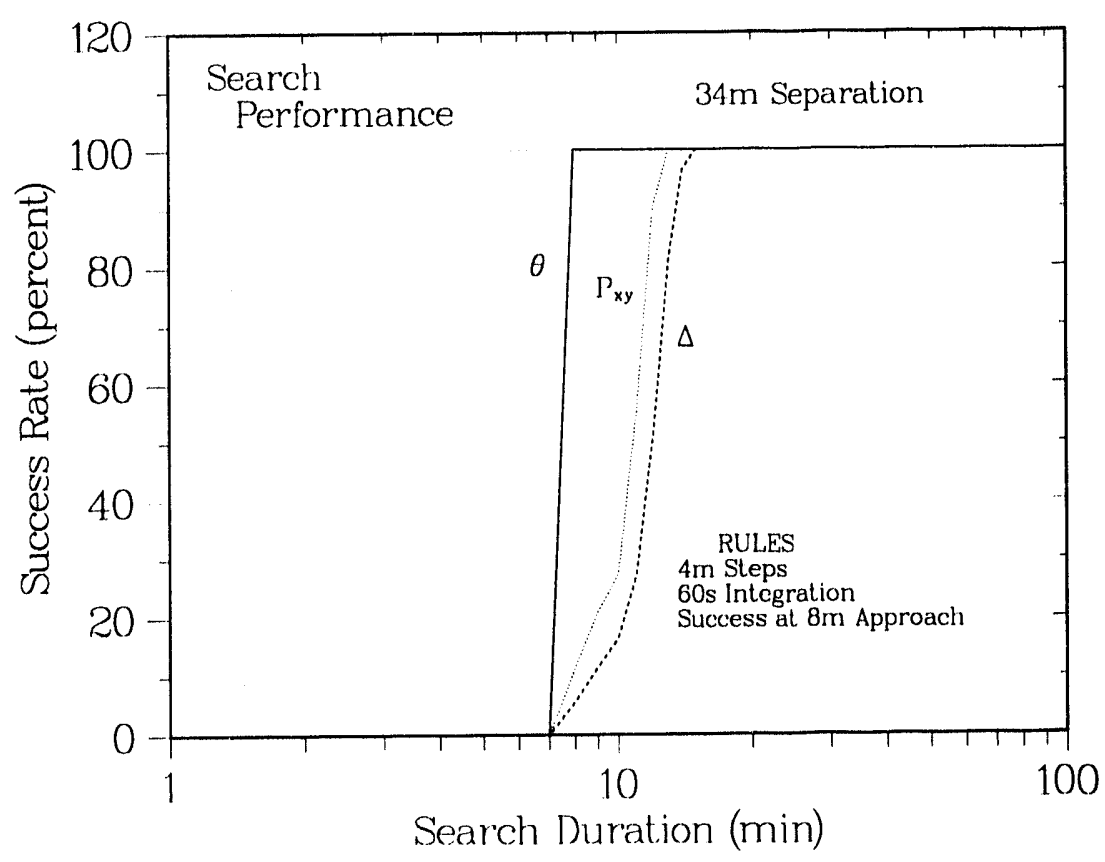

(b)

Fig. 5.2a,b. Search success rates for different strategies. At each separation distance, the probability of locating a source depends on the time allowed for the search. The upper panel (part a) is for a $10-\mathrm{m}$ source separation; the lower panel (part b) is for $34 \mathrm{~m}$. 


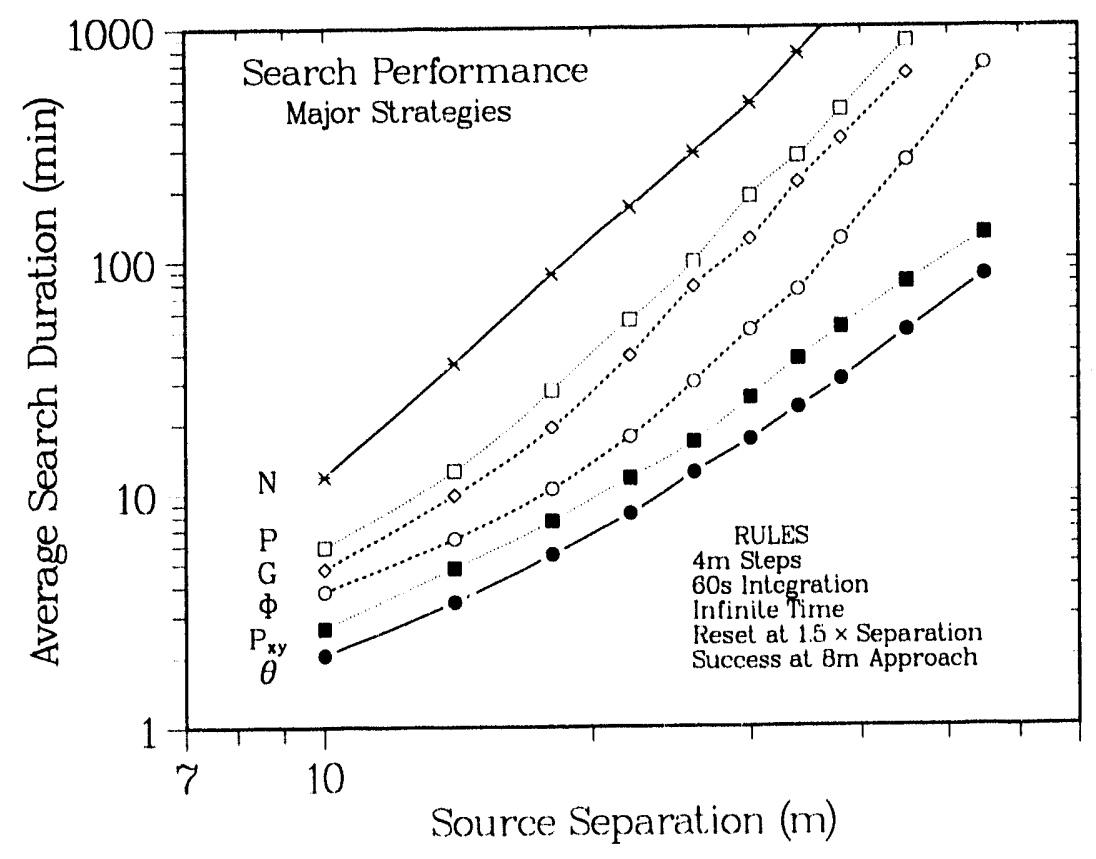

(a)

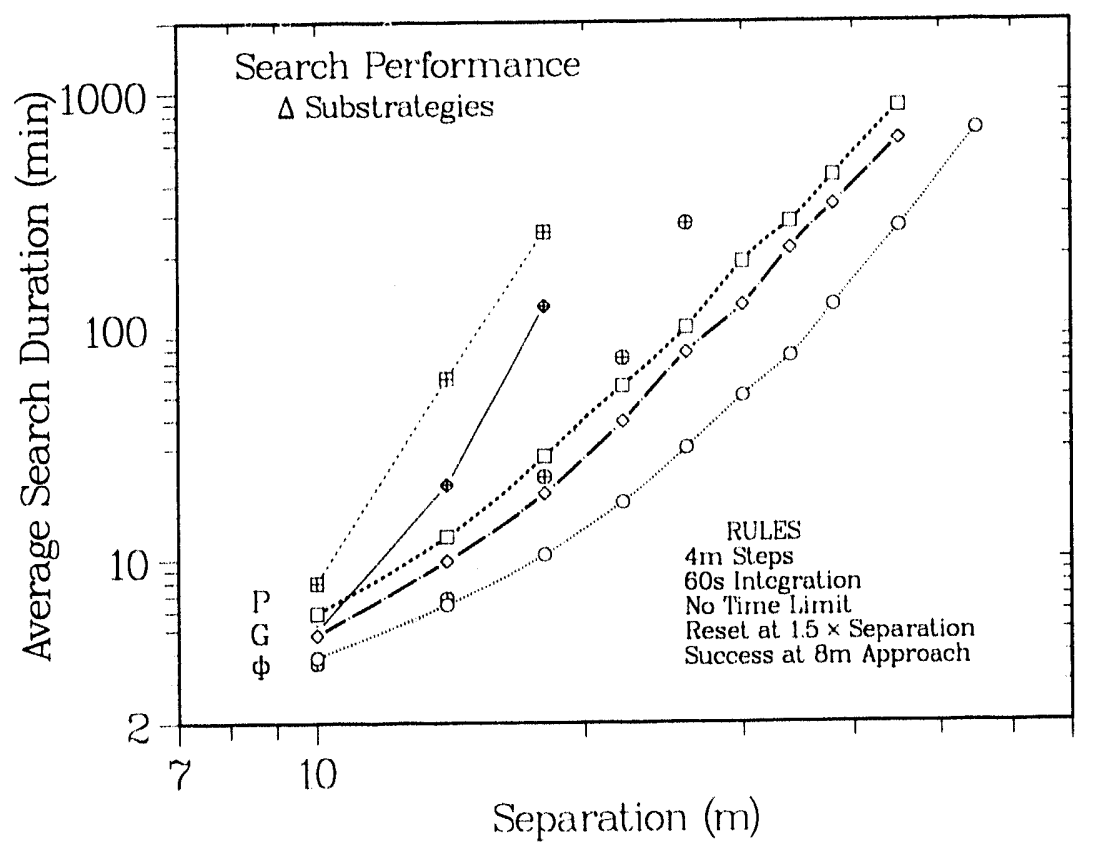

(b)

Fig. 5.3a,b. Effect of statistical uncertainties on the average search durations of Fig. 5.1. The upper panel (part a) shows the results for the major strategics; the lower panel (part b) compares two different substrategies for the cases that depend on changes between measurements at two different locations. 
result implies a power-law proportionality $\left(T \propto D^{3}\right)$ between the search time $T$ and the separation distance $D$ (the exact exponent is 3.26 ). This third-order dependence has been observed empirically in actual searches for radiation sources.

Search Substrategies. For each of the $\Delta$ strategies $(\Phi, G$, and P), two substrategies were considered. In one case, account was kept of the "absolute" best value obtained throughout the search, and decisions were referenced to changes from this value; in the other case, only the "relative" change between two immediately sequential measurements was considered. The comparisons in Fig. $\mathbf{5 . 3 \mathbf { b }}$ reveal a consistent distinction between these two substrategies. The light and heavy lines of each type indicate the two cases: the light line considers only sequential, relative changes; the heavy line references the absolute best values. For situations with good statistical accuracy (short separations), the conservative approach of the absolute comparison provides an advantage. With poorer statistics, however, this conservatism simply constrains the search to an unprofitable region and therefore greatly decreases the success rate at larger separations. Thus, the relative substrategy usually has the advantage, and its results are the ones shown in Fig. 5.3a.

Success Rates, with Statistics. The effect of statistical uncertainties on the success rates at different separations is shown in Figs. 5.4a-b. The different line types again distinguish among the different major strategies, which occur in the same order: $\theta, P_{x y}$, $\Phi, G, P$, and $N$. The contribution from counting statistics has significantly increased the differences in duration between the various strategies. Even at a reasonable $18-\mathrm{m}$ separation, achieving an $80 \%$ success rate requires at least the following search times: $\theta$, $6 \mathrm{~min} ; P_{x y}, 9 \mathrm{~min} ; \Phi, 13 \mathrm{~min} ; G, 29 \mathrm{~min} ;$ and $P, 44 \mathrm{~min}$. (For the random search, $N$, the time duration for an $80 \%$ success rate is beyond the maximum value recorded by the calculations.) Also, the different substrategies (light and heavy lines) again show the dependence on good statistics; for example, all the absolute strategies fail completely at greater than $\sim 30 \mathrm{~m}$ (see Fig. $5.3 \mathrm{~b}$ ).

Limited-Duration Searches. Inspection of the shapes of the different success-rate curves in Figs. 5.4a c suggests that achieving the highest rates requires increasingly prohibitive durations. Because searches with no time restrictions are unrealistic, an alternative constraint was used to limit the time allowed. At each separation, a successful search should reach the source in a reasonable number of steps, perhaps three times the minimum number expected for each combination of separation and step size. For example, with 4-m steps the direc: approach to a source at $20 \mathrm{~m}$ would take 5 steps; we therefore allow 15 steps, or $15 \mathrm{~min}$. Similarly, a source at a separation of $80 \mathrm{~m}$ is allowed $20 \times 3 \mathrm{~min}$, or $1 \mathrm{hr}$. This prescription provides results that allow the dependence of success rate on separation to be shown on a single plot for all strategies. Figure 5.5a shows such a plot, which compares the two substrategies for the three $\Delta$ cases and again shows that comparing with the absolute best values succeeds only at the closest, separations. Figure $\mathbf{5 . 5} \mathbf{b}$, which therefore includes only the relative substrategies for these cases, shows that an $80 \%$ success rate is achieved for the $G$ and $\Phi$ searches only for sources within separations of $10 \mathrm{~m}$ and $14 \mathrm{~m}$. For the $P_{x y}$ and $\theta$ searches, the corresponding separations can be extended to $22 \mathrm{~m}$ and $30 \mathrm{~m}$, respectively. This advantage in directionality is also supported by Fig. 5.5c, which shows the average duration of the successful searches from Fig. 5.5a. As in our first figure in this section (Fig. 5.1), the different strategies fall into three major categories, but now with differences that slowly disappear as the separation increases.

Relative Advantages. The improvements in search performance provided by the directional information are summarized in Figs. 5.6a,b, which are calculated from the results in Figs. 5.3a and 5.5b. Because a conventional search uses the intensity information provided by a single-clement detector, referred to as a $\Phi$ search throughout this section, 


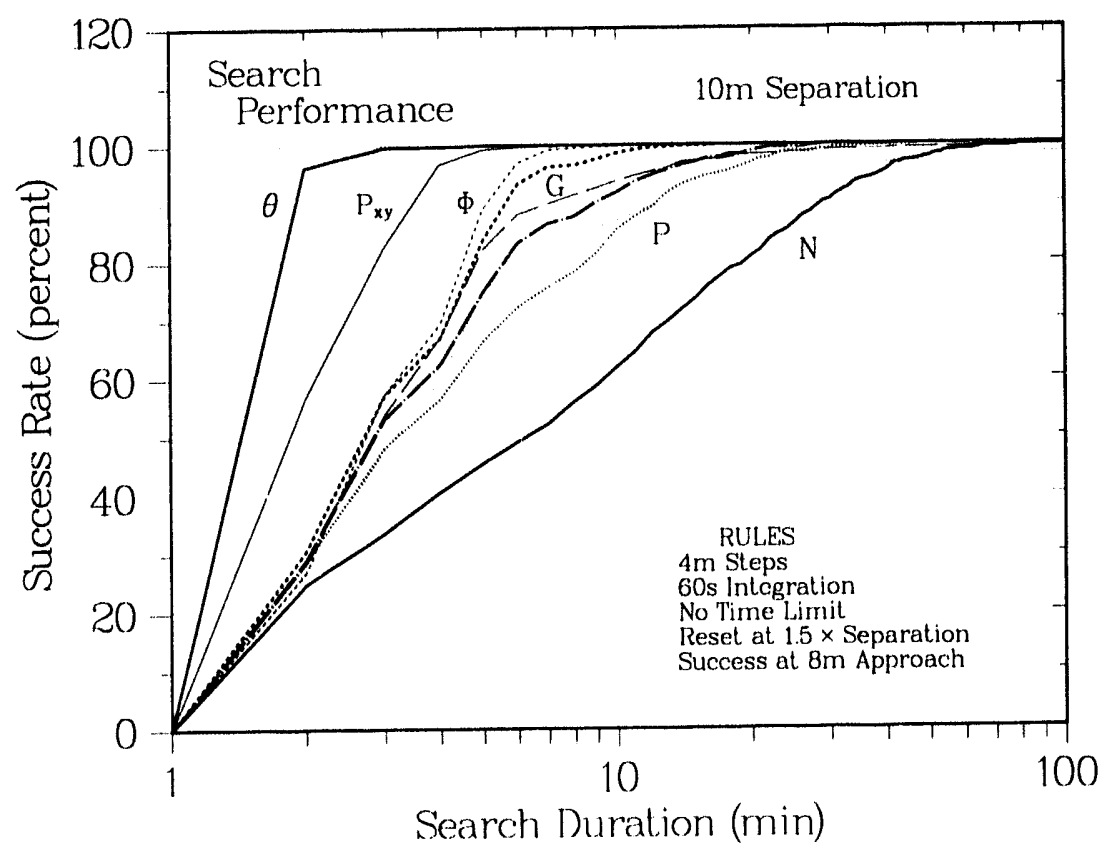

(a)

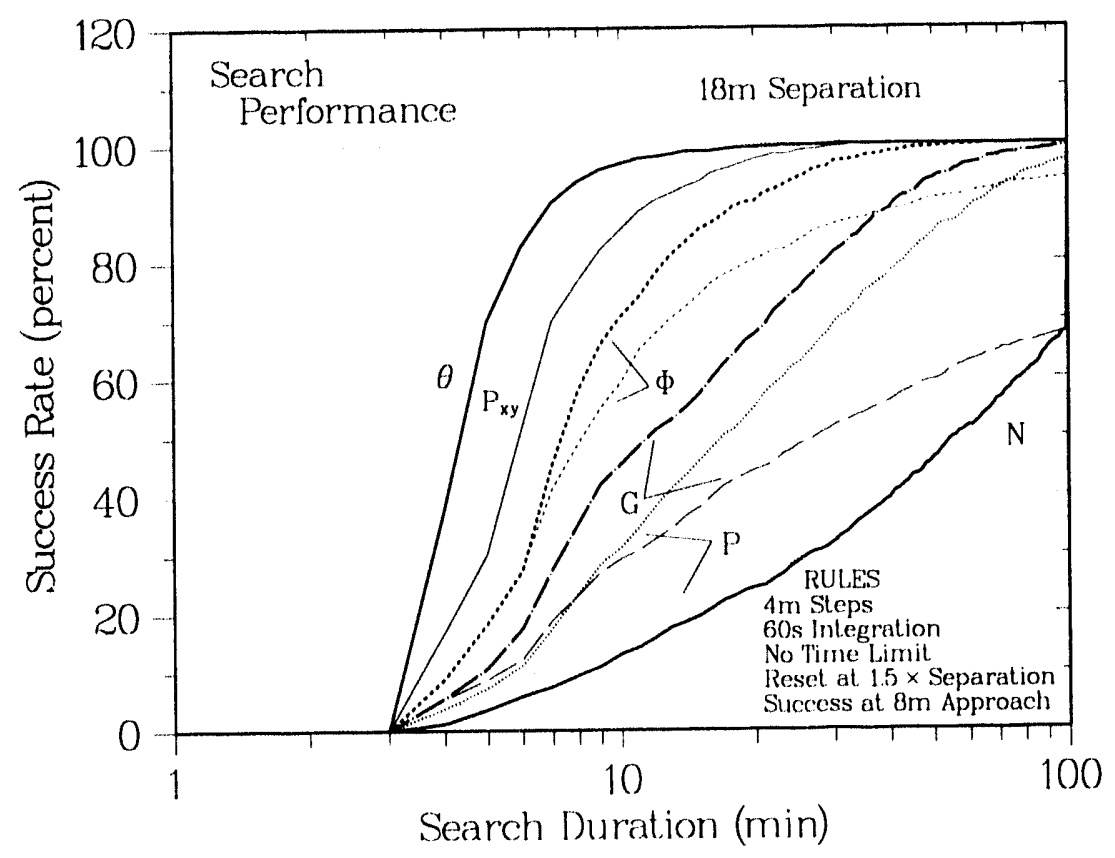

(b)

Fig. 5.4a,b. Search success rates for different strategies, including statistical uncertainties. The upper panel (part a) is for a $10-\mathrm{m}$ separation; the lower panel (part b) is for $18 \mathrm{~m}$. Compared with Fig. 5.2 (no statistics), the strategies now have diverging profiles and require longer durations. 


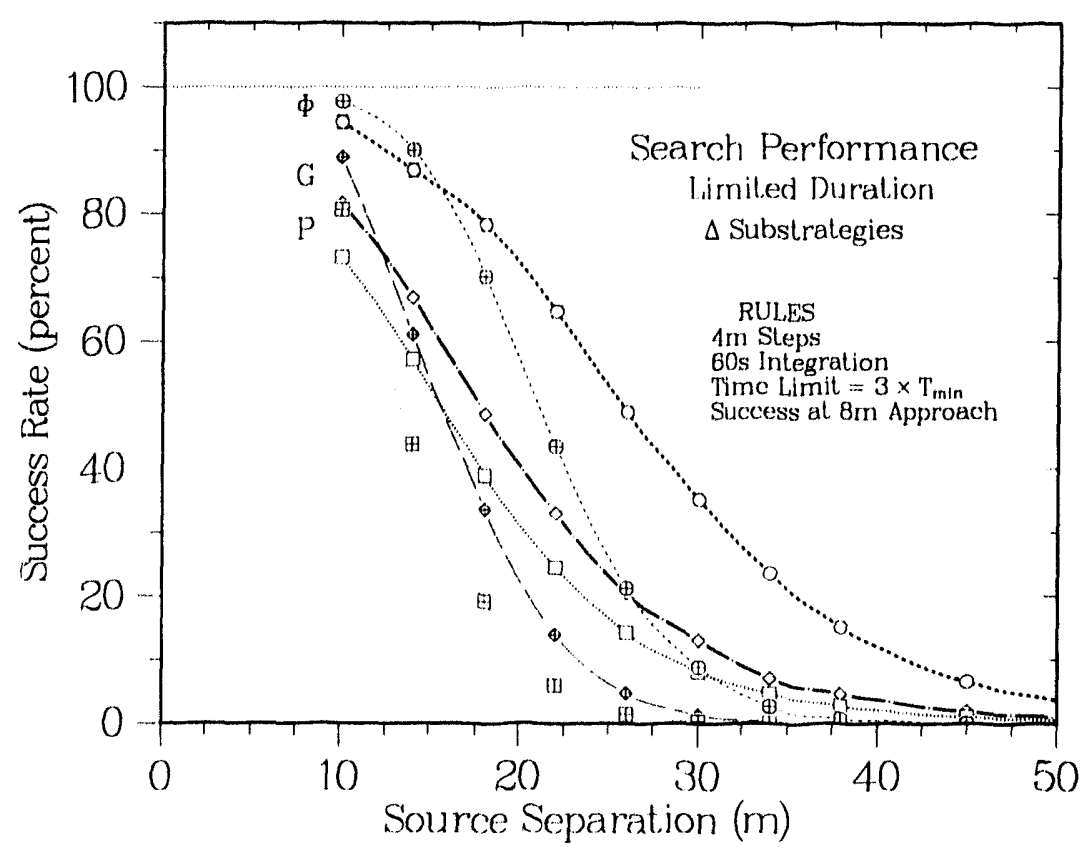

(a)

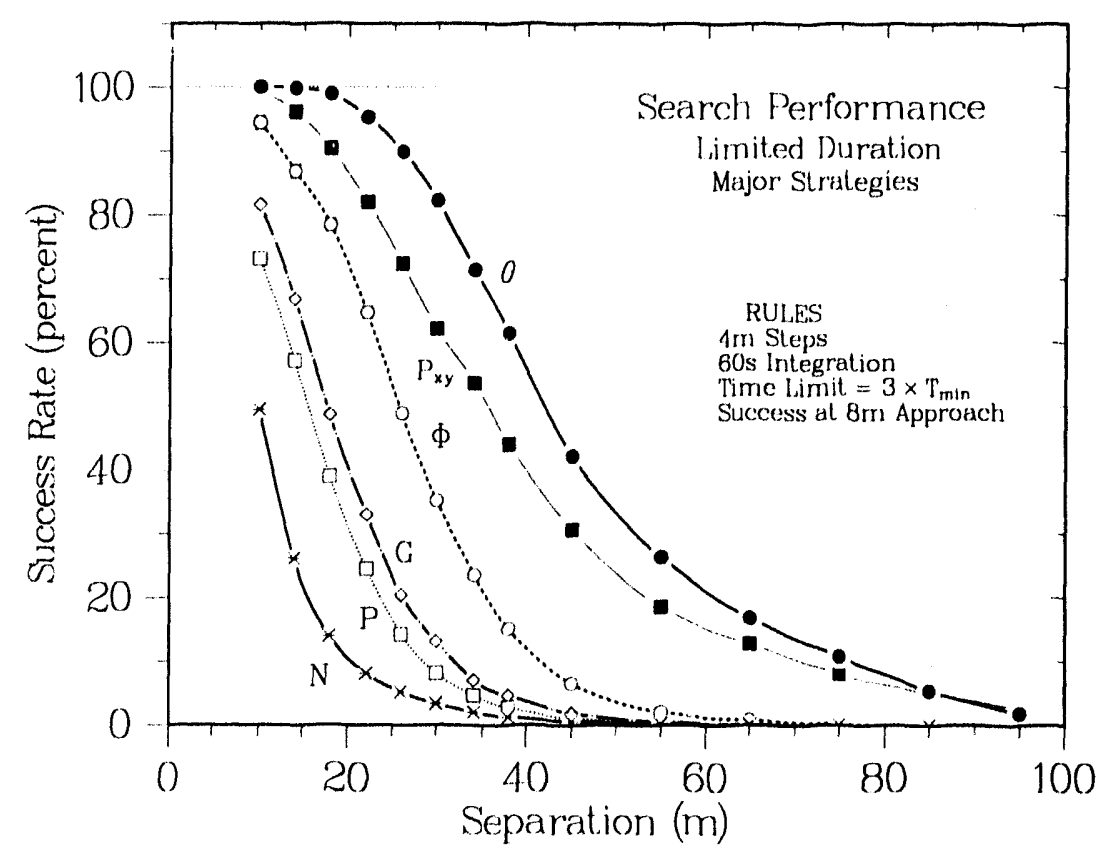

(b)

Fig. 5.5a,b. Success rates for searches with limited durations. In these cases, individual searches are required to locate the source within a prescribed time limit for each separation. 'The upper panel (part a) compares the different substrategies; the lower panel (part b) shows the results for the major strategies. 


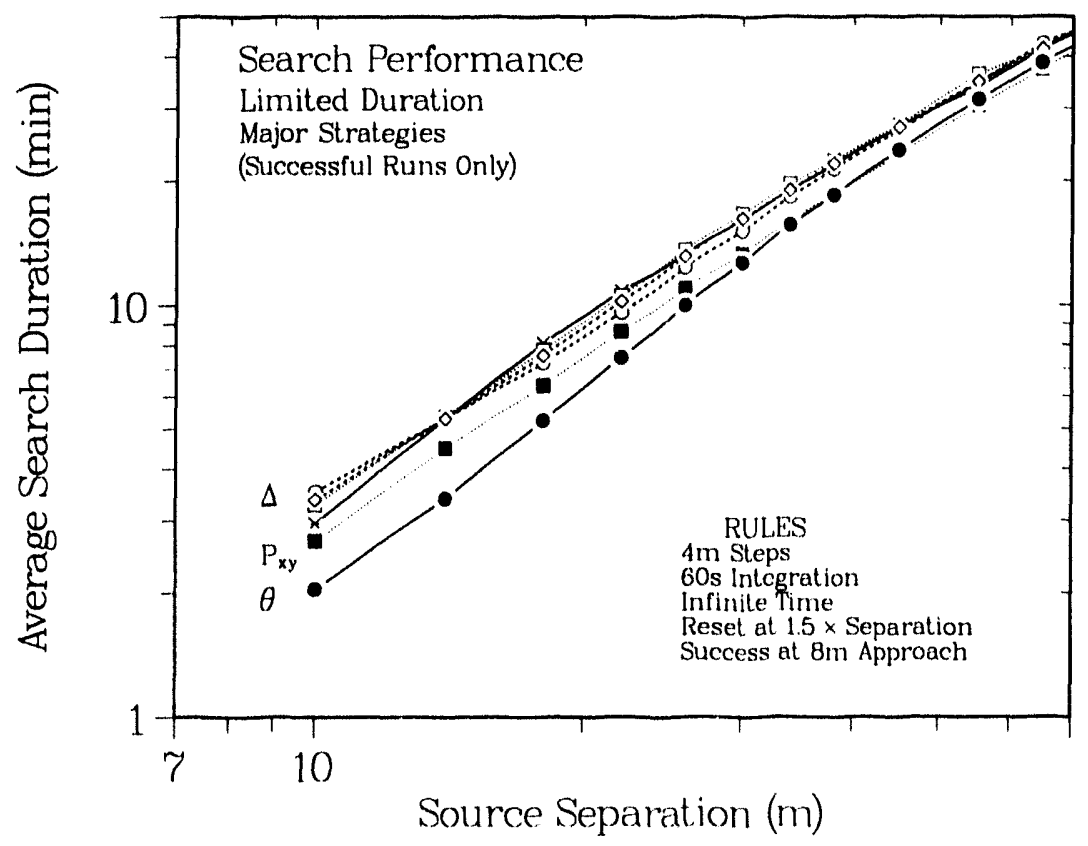

Fig. $5.5 \mathrm{c}$. Average search duration for the successful searches from Fig. 5.5b. The results fall into the same three classes as those in Fig. 5.1.

we have normalized the performance of all other strategies to this conventional case. Figure 5.6a shows the advantages in search duration, which are typically a factor of 2 or more for the most directional strategies $\left(\theta\right.$ and $\left.P_{x y}\right)$ and a factor of 0.5 or less (that is, a disadvantage) for the less directional cases $(G, P$, and $N)$. The effects increase at larger separations. Similar results are seen in Fig. 5.6b, which shows the advantages in increased separation as a function of the specified success rate. In either figure, it appears that the additional information content of the $\theta$ and $P_{x y}$ searches is a significant advantage, one that outweighs the loss in statistical accuracy caused by splitting the count rate across four elements. For the $\Delta$ cases of the $G$ and $P$ searches, however, the statistical disadvantage apparently outweighs by the smaller advantage in information content.

Summary. Although computer simulations cannot take the place of actual measurements, the calculations in this section have provided a valuable first look at the behavior of different search strategies. Considering that at each of the 14 separations, the unlimitedduration studies involved 1000 trials and the limited-duration cases included 10000 trials, the simulation approach represents the only realistic possibility for providing a quick overview of the search problem. If desired, subsequent investigations can allow even greater flexibility by including, for example, changes in parameters such as search times, step sizes, and detector areas. Such studies are especially useful because of the strongly nonintuitive behavior of searches that include randomness at different levels, such as statistical count rates and arbitrary choices of angles or starting locations. For example, the general advantage of the relative substrategy over that based on comparison with the best previous result is a statistical effect that was completely unexpected. Examining the trends from several calculations can therefore lead to important new insights, such as the central role of success rates in connecting search durations and separations. In our cases, these insights are represented by the final figures (Figs. 5.6a,b), which allow a clear comparison of the relative advantages of the various strategies in different situations. 


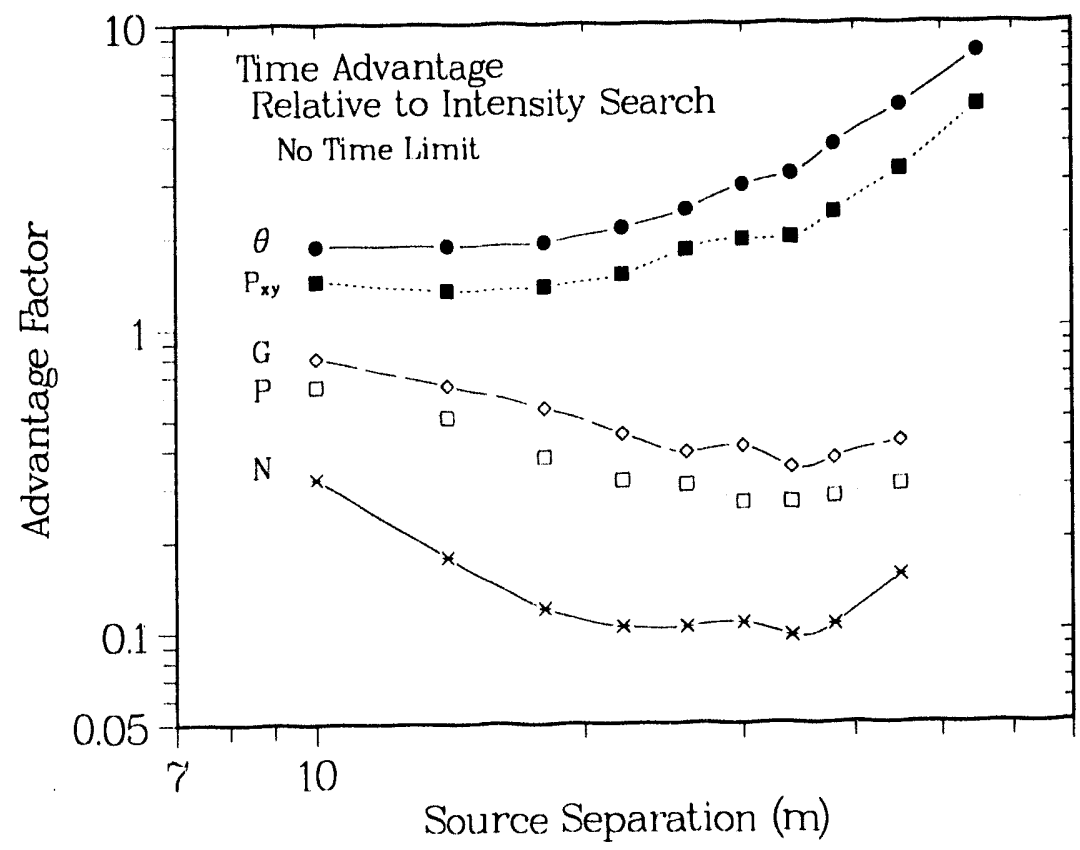

(a)

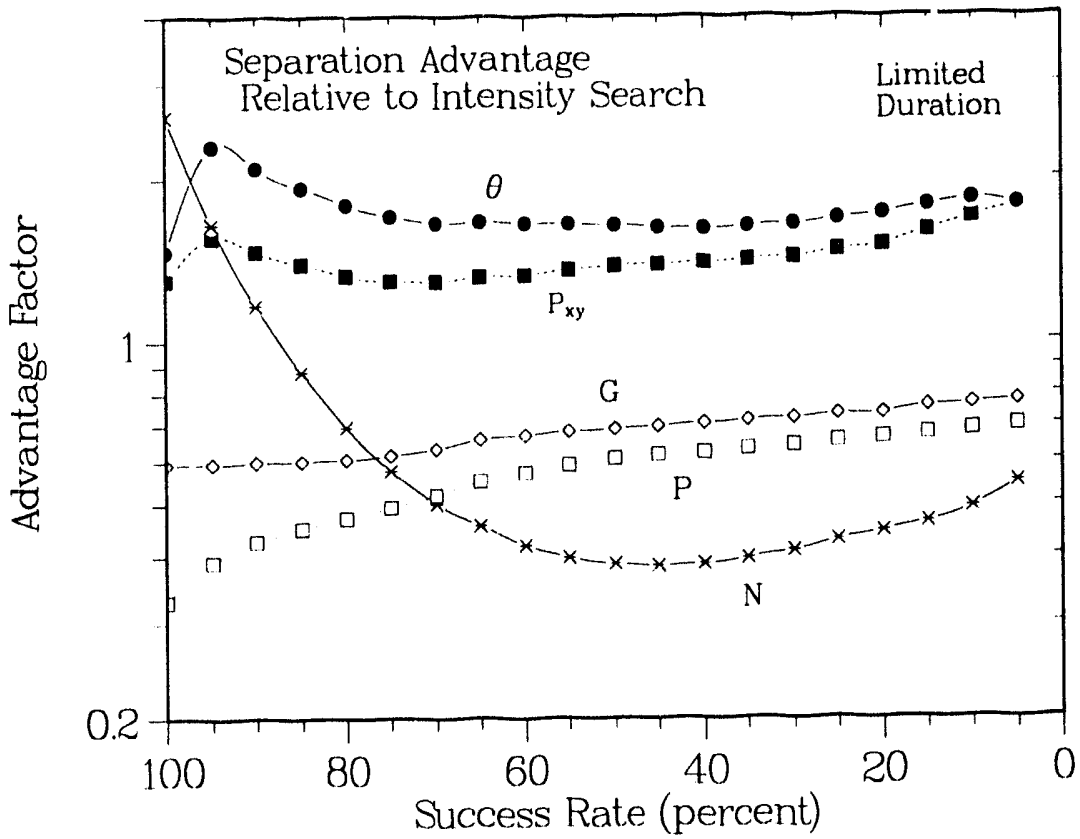

(b)

Fig. 5.6a,b. Relative advantage provided by alternate search strategies, normalized to the results for a standard intensity search $(\Phi)$. The upper panel (part a) shows ratios between average durations for searches with no time limits (from Fig. 5.3a); the lower panel (part b) shows ratios between the required separations for limited-duration searches (from Fig. 5.5b). 


\section{MISCELLANEOUS DIRECTIONALITY APPLICATIONS}

Overview. This section describes two additional examples of the type of information provided by segmented detectors. The first application exploits the relationship between source separation and polarization magnitude (see Fig. 4.2), which can be combined with the polarization direction to estimate the location of a suspected source. The second follows from the calculations in Ref. 1 for segmented neutron spectrometers, which suggest that comparisons between the spectra measured on the front and back sides of the detector can be used to estimate a background-corrected energy spectrum.

Estimated Source Locations. For simplicity, the search strategies described in the previous section make only limited use of the connection between the measured polarization magnitude and the estimated source separation, which could be used, for instance, to vary the step size or integration time. Establishing the basis for such an approach is our first application of directionality, For an initial investigation, we define an inverse polarization as $P^{\prime}=1 / P$, which has back/front $\left(P_{x}^{\prime}\right)$ and right/left $\left(P_{y}^{\prime}\right)$ components given by

$$
\begin{gathered}
P_{x}^{\prime}=P^{\prime} \cos \theta, \quad P_{y}^{\prime}=P^{\prime} \sin \theta \\
\left(d P_{x}^{\prime}\right)^{2}=\left(P^{\prime} \sin \theta d \theta\right)^{2}+\left(d P^{\prime} \cos \theta\right)^{2} \\
\left(d P_{y}^{\prime}\right)^{2}=\left(P^{\prime} \cos \theta d \theta\right)^{2}+\left(d P^{\prime} \sin \theta\right)^{2} .
\end{gathered}
$$

With this transformation, sources at "moderate" separations (comparable to the detector or source sizes) should be located near a unit circle $\left(P^{\prime}=1.0\right)$, those far enough away to produce significant scattered backgrounds should be well outside the circle, and those close enough to cause solid-angle distortion should be inside the circle. Figures 6.1a,b illustrate the results of this approach. With the detector located at $(0,0)$, the circles are plotted at the $\left(P^{\prime}, \theta\right)$ positions calculated from the inverse polarization values; the radii are statistical errors. Thus, the circles show the expected source locations and uncertainties. As expected, almost all the sources are placed directly in front of the detector; the line at $4.8 \pm 1.8^{\circ}$ indicates the average source angle. To indicate the separation information, solid circles are used for sources whose true separations are at $25 \mathrm{~m}$; dashed circles are used for more distant sources $(>5 \mathrm{~m})$, and dotted circles are for very close sources $(<2 \mathrm{~m})$. As expected, in Fig. 6.1a all the distant sources (dashed circles) have large inverse polarizations (above 1.25). An enlarged view for the nearer sources is shown in Fig. 6.1b, along with three arcs that represent the range of $A$-values expected from the calculations of Ref. 1; most of the measurements fall within these limits. The anomalous cases (dotted circles with normal $P^{\prime}$ values, solid circles with small $P^{\prime \prime}$ values) are the same ones pointed out previously in the discussion of Fig. 4.2. Thus, the combination of the polarization angle and magnitude, that is, the polarization vector, may provide a useful estimate of the source location.

Separation Distances. The next step in correlating the measurec polarization with the source location involves estimating the source separation $D$ from the inverse polarization $P^{\prime}$. Inspection of the correlation for the present data set reveals that an empirical relation is provided by

$$
D_{e}=a e^{b P^{\prime \prime}} \text {, }
$$

with $a=1500 \mathrm{~cm}$ and $b=2.8$ for the present data. The results are shown in Fig. 6.2 , which plots the estimated separation $D_{e}$ versus the actual source distance ( $D$, given by DIST for the cases in Table I). With the exceptions described near the end of Sec. 4, the agreement between the true and estimated separations is convincing. Although our particular parameters and functional form may be accurate only for the present measurements, a similar approach should be appropriate in other situations. 


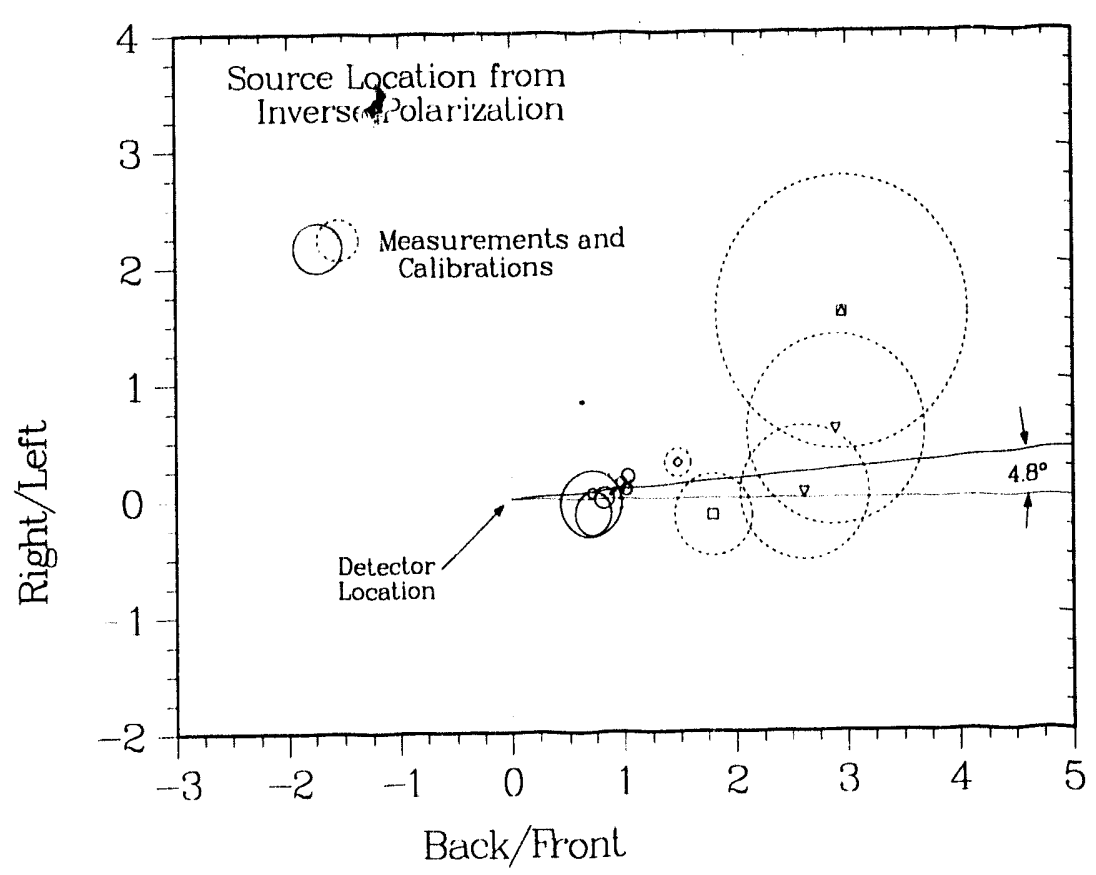

(a)

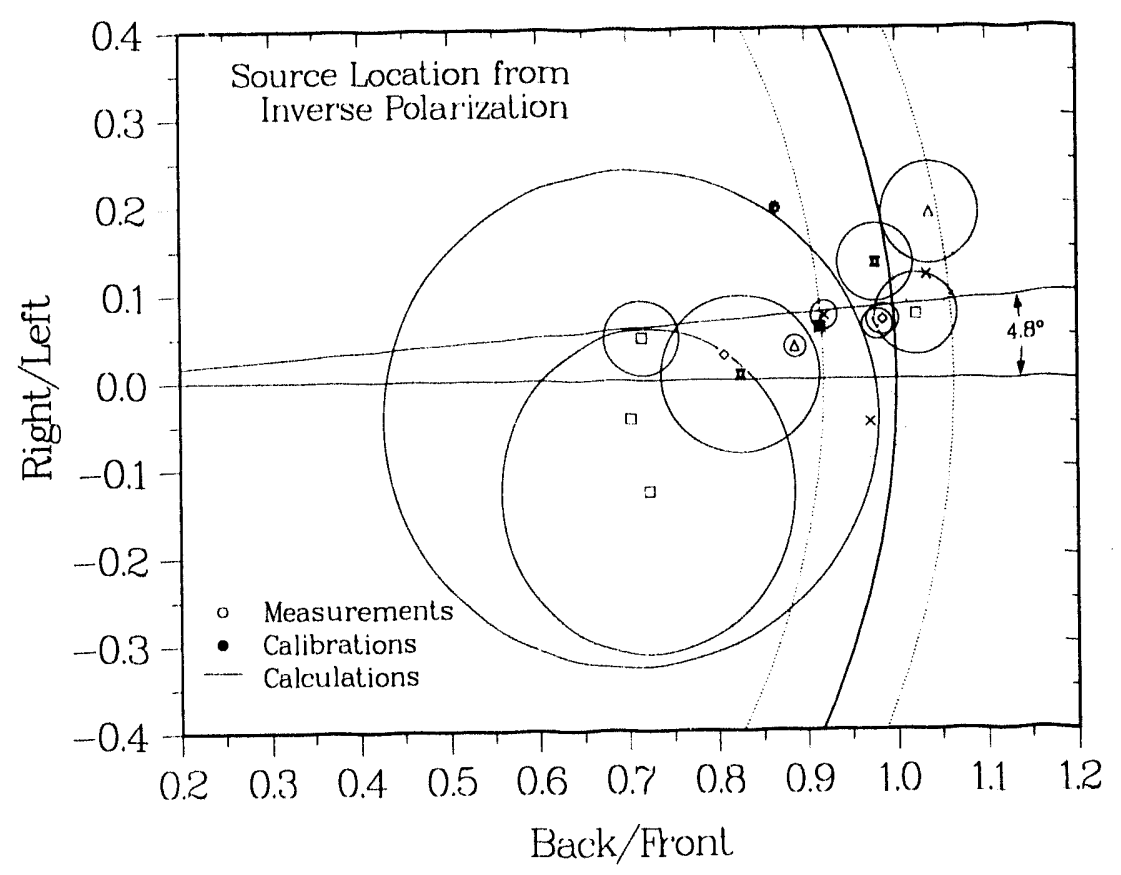

(b)

Fig. 6.1a,b. Upper panel (part a): Estimated source locations from inverse polarization values. The source angles are obtained from the vector directions, and the estimated separations are from the inverse magnitudes of the polarizations. The different circles indicate the statistical errors. The lower panel (part b) is an enlarged section of part (a). 


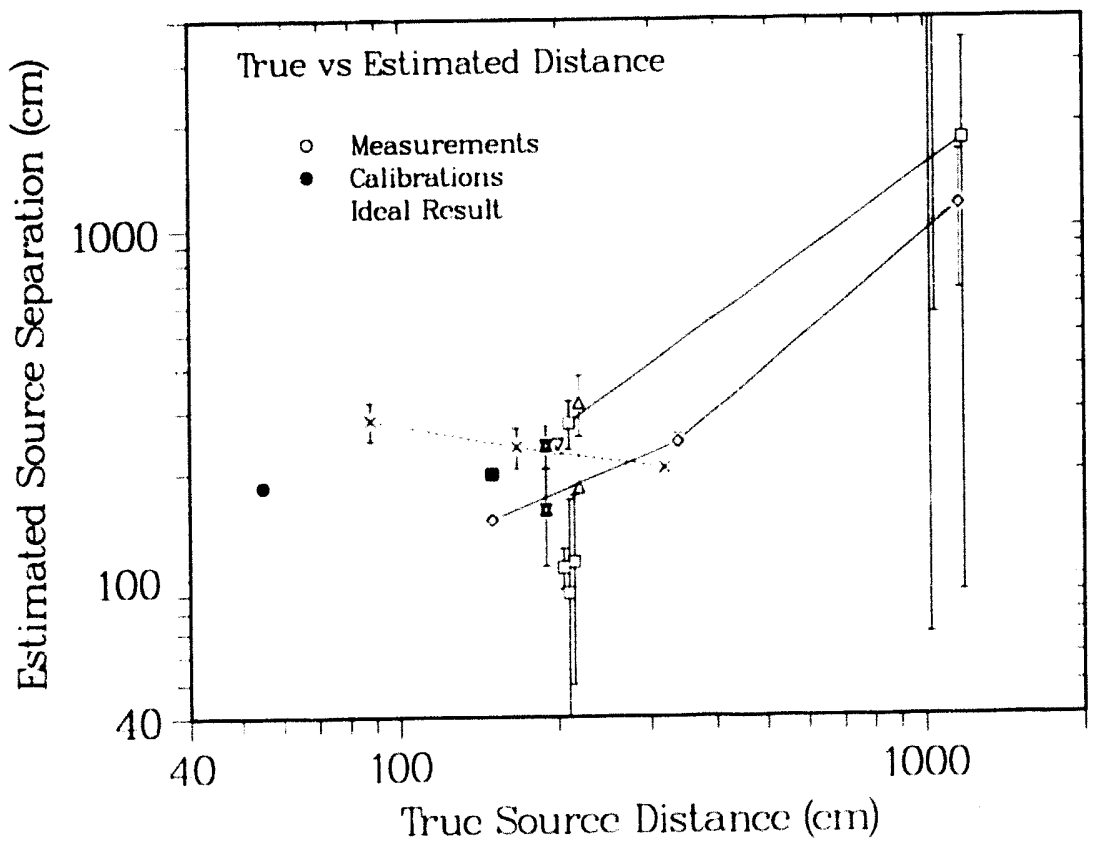

Fig. 6.2. Correlation between the true source separation and that estimated from the measured inverse polarization using an empirical relation.

Background Corrections. The second application of segmented detectors is their use for estimating background corrections for the measured energy spectra described in Ref. 6. In this application the detector is divided into pairs of front and rear rods, which should include different amounts of directional signal and scattered background. Several approaches seem to be reasonable. The simplest calculation, a front-rod spectrum, uses only the two rods facing the source and neglects those facing the opposite direction. Next, an asymmetry approach exploits the polarization argument to assert that the largest $R$ values (near 0.8 ) correspond to fully directional fluxes with no scattered background (as for a nearby source), while $R=0$ suggests an omnidirectional scattered flux (as for a very distant source). In an attempt to avoid restriction to our particular situation (and also serious oversubtraction for nearby sources with $R \sim 0.8$ ), we have expressed the dependence in terms of the asymmetry $R$, not the polarization $P$. Scaling the average front-rod flux by a factor $f_{a}=(R / 0.8)^{1 / 2}$ gives an estimated correction

$$
\phi_{a}=f_{a}\left(\phi_{1}+\phi_{2}\right) / 2 .
$$

Additional studies will be needed to test the accuracy of this empirical prescription.

One-Sided Corrections. Next, there are two one-sided approaches discussed in Ref. 1, which use either energy-independent or energy-dependent corrections. Th underlying assumption is that a scattered flux is mainly incident on the detector from the rear and leaks through into the front rods. The more general energy-dependent correction proceeds as follows. As the neutron energy increases, so does the transmission of background through the detector from rear to front. The Monte Carlo calculations in Ref. 1 suggest transmission values of $0.22,0.33,0.44$, and 0.55 at $1,2,4$, and $8 \mathrm{MeV}$; when fitted, these values give the function

$$
B_{f}=f(E) \phi_{r}=0.11\left(\log _{2} E\right)\left(\phi_{3}+\phi_{4}\right) / 2 .
$$


The background-subtracted front-rod yield is then

$$
\phi_{s}=\phi_{f}-B_{f}=\left(\phi_{1}+\phi_{2}\right) / 2-B_{f} \text {. }
$$

As shown in Ref. 1, this subtraction reduces the front-rod detection efficiency by fractions of $0.01,0.074,0.138$, and 0.296 at the four energies, which can be corrected by dividing $\phi_{s}$ by $g(E)=1.02-0.038 E$, that is, the background-corrected front-rod yield $\phi_{1 e}$ is

$$
\phi_{1 e}=\left(\phi_{f}-f(E) \phi_{r}\right) / g(E) \text {. }
$$

The more limited energy-independent case proceeds in the same way, but substitutes constant values $f(E)=0.5$ and $g(E)=0.78$ :

$$
\phi_{1 c}=\left(\phi_{f}-0.5 \phi_{r}\right) / 0.78 \text {. }
$$

Transmission Corrections. The two final transmission corrections are similar to the one-sided approach, except the detector is assumed to be subject to a strong directional signal flux with a weak omnidirectional scattered background. A known fraction of the directional flux is transmitted from the front into the rear rods; subtracting this predicted leakage from the measured rear-rod flux provides an estimate of the assumed isotropic background, which can in turn be subtracted from the front-rod flux. In this approach the transmitted flux, the isotropic background, and the corrected yield are:

$$
\begin{gathered}
T_{r}=f(E)\left(\phi_{1}+\phi_{2}\right) / 2 \\
B_{r}=\left(\phi_{3}+\phi_{4}\right) / 2-T_{r} \\
\phi_{t e}=\left(\phi_{f}-B_{r}\right) .
\end{gathered}
$$

Again, the energy-independent case substitutes a constant transmission value:

$$
\begin{gathered}
T_{r}=0.5\left(\phi_{1}+\phi_{2}\right) / 2 \\
B_{r}=\left(\phi_{3}+\phi_{4}\right) / 2-T_{r} \\
\phi_{t c}=\left(\phi_{f}-B_{r}\right) .
\end{gathered}
$$

Average Correction. Each of these six approaches gives a reasonable background distribution. We have therefore averaged the results together and added their variance to the statistical error to estimate an overall systematic uncertainty. Because the front-rod (no-correction) and one-sided approaches are supported by previous studies, these results were given more weight in the averaging. Figure 6.3 shows the corrected spectrum $\phi_{a}$ obtained for the ${ }^{252}$ Cf calibration run. As discussed in Ref. 6, the agreement with the expected fission distribution is very good at all energies above the detector threshold near $1 \mathrm{MeV}$. For this particular case, the background-subtracted result is almost indistinguishable from the original front-rod spectrum, both in shape and magnitude. In fact, the correction increases the area by about $1.3 \%$; this increase is within the generated uncertainty, which has 


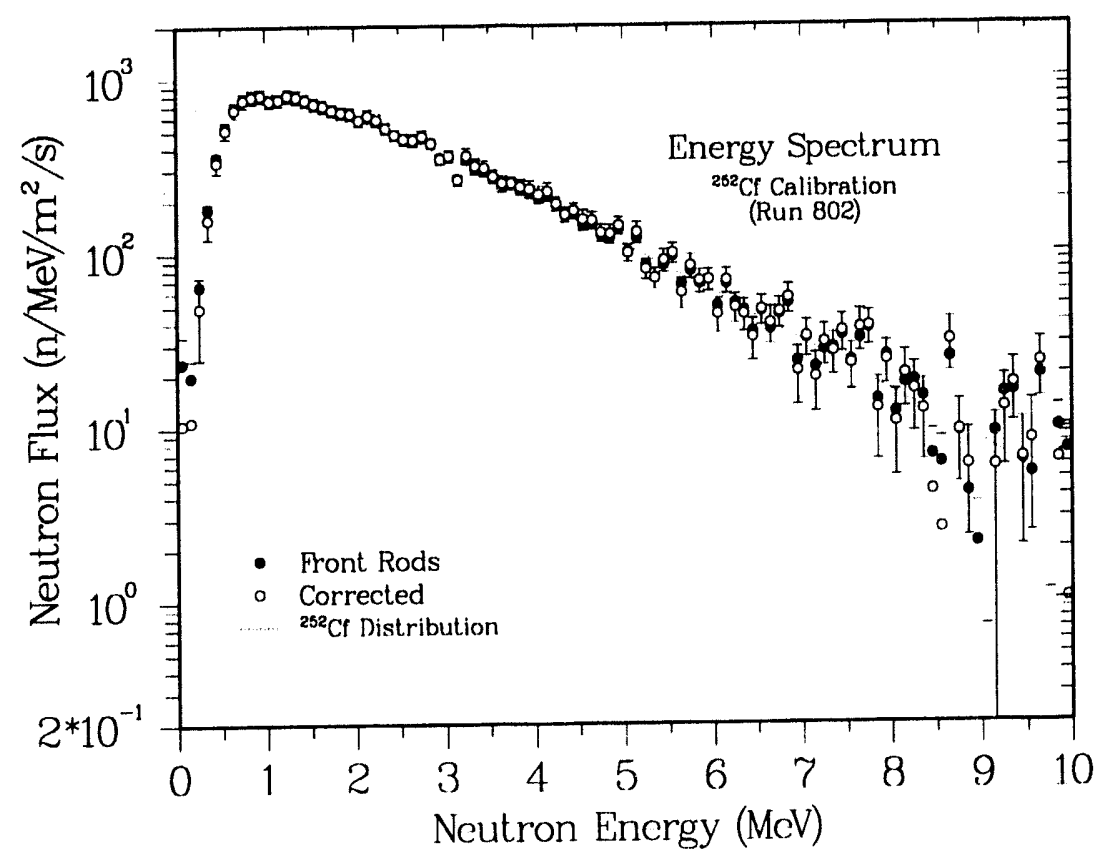

Fig. 6.3. Front-rod and background-corrected energy spectra for the ${ }^{252} \mathrm{Cf}$ calibration run. Because the source was located only $54 \mathrm{~cm}$ in front of the detector, the background correction is negligible. (Errors are shown on the corrected data only.) The ${ }^{252} \mathrm{Cf}$ curve is an evaluation from Ref. 7 .

increased to $1.3 \%$ from a statistical error of $0.5 \%$. This conservative correction is reasonable in view of the relatively favorable geometry used for the calibration measurement.

Background Systematics. 'To summarize our background discussion, Fig. 6.4 shows the observed relationship between the signal/total ratios $S / T$ and the measured asymmetries $R$ from all the data. As expected, lower $R$-values correspond to larger background estimates and lower $S / T$ values. Most of the measurements are clustered in a narrow region near the calculated range of $R$-values. The $S / T$ ratios for some runs (including the calibrations) are slightly greater than 1.0 , because some source separations are so small that solid-angle effects lead to high asymmetries. The dashed line shows the empirical function $S / T=1.1 R^{1 / 4}$, which is constrained to pass through the predicted value of 1.0 at $R=0.62$. Note that the lowest $S / T$ ratios of $0.8-0.9$ correspond to $S / B$ values of about $6: 1$, which are much higher than the 1:1 ratio estimated in Secs. 3-4. This difference is associated with the wider range of background estimates included in the average value obtained in this section.

Discussion. The two applications described in this section are based on the polarization analyses in Sec. 4 and the earlier calculations of Ref. 1. Both discussions are constrained by the limited range of variation in the present measurements, which were not originally intended as a study of the detector's directional capabilities, and additional experiments will be needed to examine the reliability of the different techniques. 


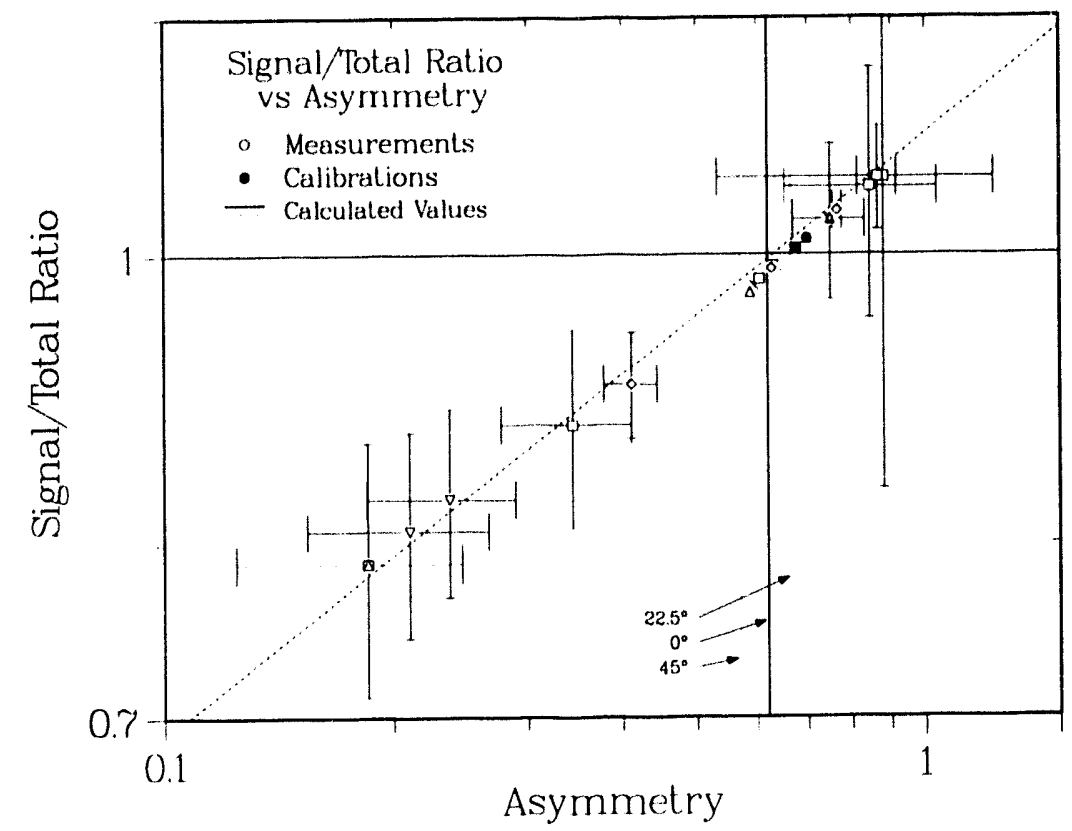

Fig. 6.4. Relationship between the average Signal/Total ratios and the measured detector asymmetries. The dashed line gives zero signal at $R=0$ and zero background at $R=0.62$. (Errors on the points near $R=0.6$ are suppressed for clarity.)

\section{CONCLUSIONS}

Polarized Fields and Directional Detectors. By combining the basic directional concepts introduced in Ref. 1 with a limited set of measurements for realistic neutron sources, this report has developed an understanding of the advantages of directional measurements for the location of sources of nuclear radiation. After the brief review of the basic detector operations in Sec. 2 , Sec. 3 introduces the concepts of polarized radiation fields and directional radiation detectors, which provide a novel approach to the critical search problem of distinguisling between signal and background. In both the present approach and in conventional techniques, it is the gradient of the radiation field that provides the source signature. In a conventional approach, measurements at different locations, with the attendant differcuces in background rates, are used to determine this signature; our approach measures the local gradient at a single position and therefore eliminates much of the background problem.

Mathematical Formalism and Experimental Demonstrations. Our concept of a. polarized radiation field leads to two important predictions that connect the polarization direction with the source angle and the polarization magnitude with the local signal-tobackground ratio, and hence the source separation. In Sec. 4, we develop the techniques for expressing these concepts in terms of measurable quantities by adapting the formalism used for polarization observables, and we provide the experimental verification of the predictions by taking advantage of an existing prototype detector and the data sets from previous experiments. With this foundation we can readily demonstrate angle accuracies within $\pm 5^{\circ}$ for both point sources and distributed objects. In Secs. 4 and 6 we also show that the polarization magnitude not only changes with increasing source separation; its 
value may even provide a reasonable estimate of the distance to the source. As with the background corrections, however, the quantitative investigation of many of these issues can be improved significantly with further measurements that are specifically intended to address the directional characteristics. Finally, the Monte Carlo simulations of Sec. 5 provide an intriguing first look at the relative advantages of directional approaches over conventional search techniques; the directional approaches appear to improve either the search range or time duration by about a factor of two.

Further Work. Three additional studies are underway to complete the analysis of the mockup measurements and address issues raised in the present report. First, a subsequent paper will discuss the comparison between the individual measurements and computer simulations of the exprected emission spectra. Second, we stress that most of the directional techniques developed in this report apply equally well to any detector of neutral radiation, including gamma-ray instruments or the polyethylene-shielded ${ }^{3} \mathrm{He}$ counters commonly used for neutron counting. For example, by simply replacing the single ${ }^{3}$ He tube with four separate tubes, it may bo possible to obtain both directional information and the standard neutron flux. Finally, the spectroscopic and directional capabilities of the present detector can in principle be combined. A single four-rod instrument could automatically use the local intensity and polarization to determine whether a source is present, use the calculated source angle to select the likely "front/back" detector orientation, estimate the background correction to the measured neutron spectrum, and then analyze the corrected spectrum in terms of the shape parameters of a standard fission distribution. All of these functions could be performed in real time using the same set of four single-rod spectra, just as demonstrated

by the analyses in this directional report and its companion spectroscopic study. ${ }^{6}$ Such an instrument should be applicable to a wide range of radiation-detection problems.

\section{ACKNOWLEDGMENTS}

These measurements were made possible with the assistance of LANL's Advanced Nuclear Technology (iroup. Marilyn Halbig of Space Data Systems adapted the spacecraft software for the backup instrument used here. This work was supported by the Field Neutron Spectrometer project, which is funded by the Office of Research and Development in the U.S. Department if Energy's Office of Intelligence and National Security.

\section{REFERENCES}

1. R. (C. Byrd, "Dirertional Fast-Neutron Detectors," Jos Alamos National Laboratory report LA-12379-NIS (October 1992).

2. W. (C. Feldman. (i. F. Auchampaugh, and R. (. Byrd, "A Novel Fast Neutron Detector for Space Applications," Nuclear Instruments and Methods A306 350-365 (1991).

3. C. E. Moss, R. (. Byrd, C. F. Auchampaugh, W. C. Feldman, C. P. Estes, R. I. Ewing, and K. W. Marlow. "Detection of Uranium-Based Nuclear Weapons Using NeutronInduced Fission." (nubmitted to IEEE Transactions on Nuclear Srience).

4. R. C. Byrd. (i. F. Anchampangh, (. E. Moss, and W. ('. Feldman, "Warhead Counting Using Neutron Scintillators: Detector Development, Testing, and Demonstration," IEEE Transactions on Nirlerar Science NS-39, 1051 1055) (August 1992).

5. Paul Whalen et al.. "Measurements and (alculations of Neutron Energy Spectra for the Radiation Environmments Program," Jos Alamos National Laboratory report (in preparation).

6. Roger Byrd, (irorg" Auchampaugh, and William Feldman, "Measurement and Analysis of Neutron Energy Spectra from Fission Sonrees." Los Alamos National Laboratory report LA-1262i-MS (October 1993).

7. F. H. Fröhner, "Ewaluation of 252 (' Prompt Fission Neutron Data from 0 to $20 \mathrm{MeV}$ by Watt Spectrum Fis." Nuclear Science and lingines ring 106, 3.15) 352 (1990). 

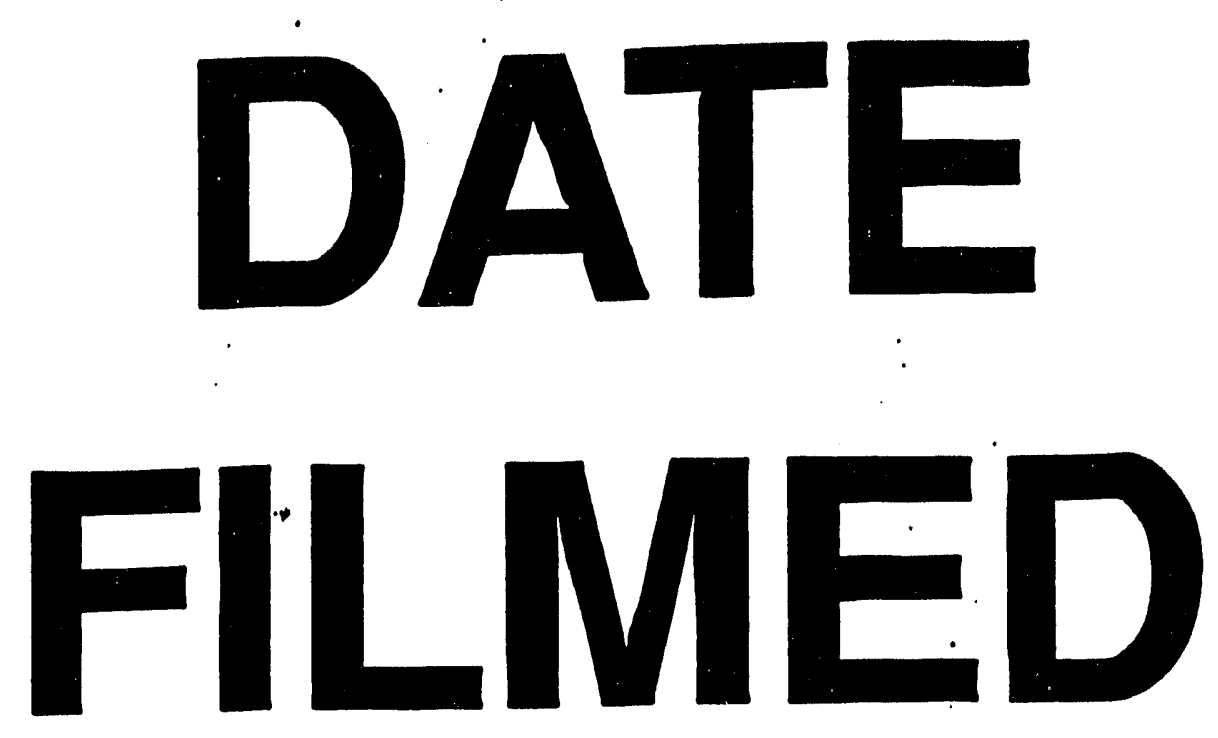

$2 / 1 / 94$
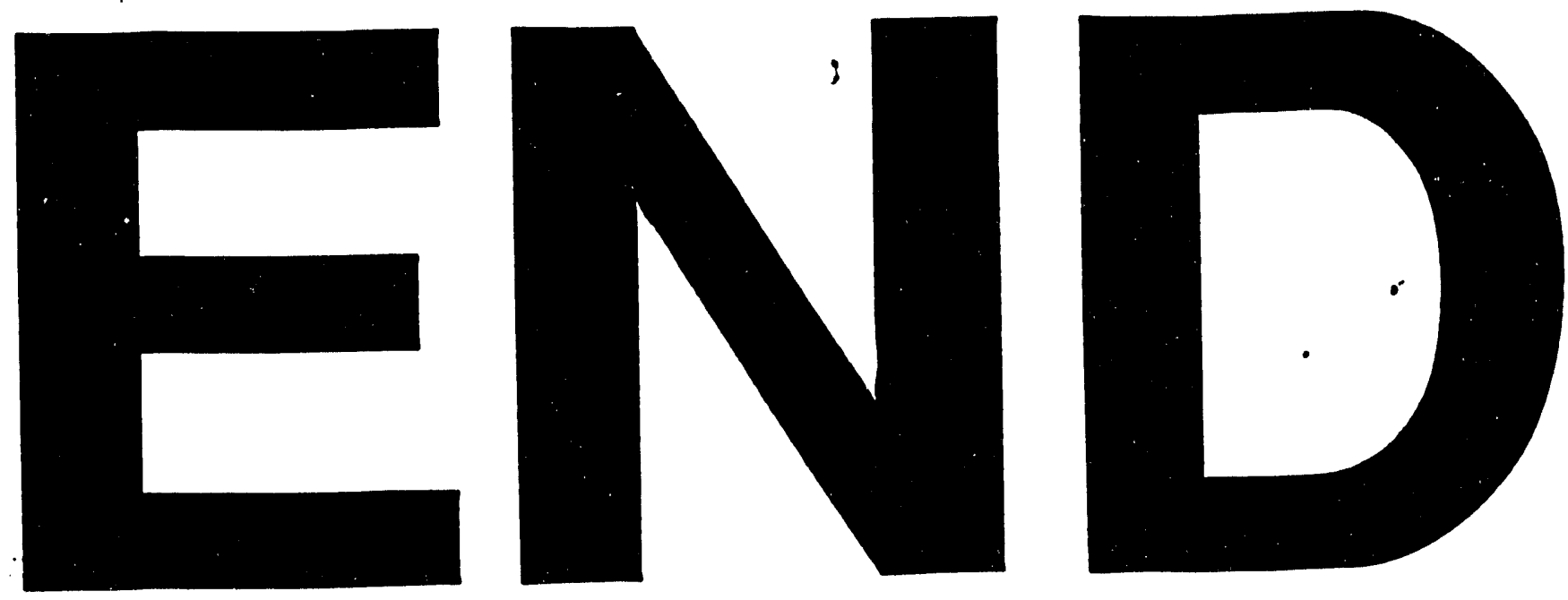


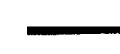

